Homology, Homotopy and Applications, vol.14(1), 2012, pp.33-63

\title{
SEMICOVERINGS: A GENERALIZATION OF COVERING SPACE THEORY
}

\author{
JEREMY BRAZAS
}

(communicated by Donald M. Davis)

\begin{abstract}
Using universal constructions of topological groups, one can endow the fundamental group of a space with a topology and obtain a topological group. Additionally, the fundamental groupoid of a space becomes enriched over Top when the homsets are endowed with similar topologies. This paper is devoted to a generalization of classical covering theory in the context of these constructions.
\end{abstract}

\section{Introduction}

The classical theory of covering spaces may be stated conveniently as the following categorical equivalence. For a locally path connected, semilocally 1-connected space $X$, the fundamental groupoid functor $\pi$ provides an equivalence of categories

$$
\pi: \operatorname{Cov}(X) \rightarrow \operatorname{CovMor}(\pi X)
$$

from the category of coverings of $X$ to the category of covering morphisms of $\pi X$ : See [6, Chapter 10] or [23, Chapter 3]. It is a standard and useful fact that the category $\operatorname{CovMor}(\mathcal{G})$ of covering morphisms of a groupoid $\mathcal{G}$ is equivalent to the functor category $\operatorname{Fun}(\mathcal{G}, \operatorname{Set})$, i.e., the category of operations of $\mathcal{G}$ on sets. Thus a covering $p: Y \rightarrow X$ is also characterized by its corresponding monodromy functor $\pi X \rightarrow$ Set. This correspondence, between structured maps of spaces and morphisms of an algebraic object, has significant application in both algebra and topology.

Generalizations of the notion of covering map and extensions of covering theoretic techniques to spaces beyond those in the classical theory have appeared in many different contexts $[\mathbf{2}, \mathbf{5}, \mathbf{1 3}, \mathbf{1 4}, \mathbf{1 7}, \mathbf{2 2}]$. In this paper, we define the notion of a semicovering map and construct a topologically enriched fundamental groupoid functor $\pi^{\tau}$ intimately related to universal constructions of topological groups. The main result is that if $X$ is what we call a locally wep-connected space, $\pi^{\tau}$ provides an equivalence of categories

$$
\pi^{\tau}: \operatorname{SCov}(X) \rightarrow \operatorname{OCovMor}\left(\pi^{\tau} X\right)
$$

from the category of semicoverings of $X$ to the category of open covering morphisms

This research was supported in part by a Dissertation Year Fellowship from the University of New Hampshire Graduate School.

Received August 16, 2011, revised October 6, 2011; published on April 26, 2012. 2000 Mathematics Subject Classification: 57M10, 55Q52.

Key words and phrases: covering map, groupoid, topological fundamental group, topological group. Article available at http://intlpress.com/HHA/v14/n1/a3 and doi:10.4310/HHA.2012.v14.n1.a3 Copyright (C) 2012, International Press. Permission to copy for private use granted. 
of $\pi^{\tau} X$. For any topologically enriched groupoid $\mathcal{G}, \operatorname{OCovMor}(\mathcal{G})$ is equivalent to the enriched functor category $\operatorname{TopFunc}(\mathcal{G}$, Set) which one is encouraged to think of as the category of continuous $\mathcal{G}$ actions on discrete sets. Here Set is enriched by giving sets of functions between discrete sets the topology of point-wise convergence. Thus monodromy, in the classical sense, is now replaced by enriched monodromy functors $\pi^{\tau} X \rightarrow$ Set. Our classification of semicoverings includes the above equivalence, the classification of coverings of arbitrary locally path connected spaces in terms of socalled "Spanier groups" $[\mathbf{1 2}, \mathbf{2 5}]$, and Fox's fundamental theorem of overlays $[\mathbf{1 4}, \mathbf{2 1}]$ as special cases.

The author emphasizes the assessment in the introduction of [13] that the properties one should require of a "generalized covering map" depend on the intended application. A semicovering map $p: Y \rightarrow X$ is a local homeomorphism such that whenever $p(y)=x$ and $f$ is a path starting at $x$ or a homotopy of paths starting at $x$, there is a unique lift $\tilde{f}_{y}$ of $f$ starting at $y$. Moreover, we demand that each lifting assignment $f \mapsto \tilde{f}_{y}$ for both paths and homotopies is continuous with respect to the compact-open topology on function spaces. Except for local triviality, semicoverings enjoy nearly all of the important properties of coverings; in this sense, the notion of semicovering is quite close to the notion of covering. The intentions of this generalization are to identify a suitable notion of generalized covering whose theory applies to spaces with non-trivial local structure, develop geometric tools for studying topological groups much like those used in applications of covering space theory to group theory [18, Ch. 14], and to better understand the topologized fundamental group $\pi_{1}^{\tau}$ introduced in [4].

Covering-type theories applicable to non-locally path connected spaces are less prevalent due to the existence of troubling cases such as Zeeman's example [19, Example 6.6.14] of a planar set which admits non-equivalent coverings that induce equivalent covering morphisms. Lubkin's theory of coverings [22] overcomes this obstacle using a more general notion of "space" and "group." The authors of $[\mathbf{1}, \mathbf{1 7}]$ achieve a quite general theory by attaching extra data (equivalence classes of locally constant presheaves) to their projection maps and providing a classification in terms of the fundamental pro-groupoid. Conveniently, semicoverings are genuine maps of topological spaces and can be classified for spaces in the class of locally wep-connected spaces. This class contains all locally path connected spaces and "enough" non-locally path connected spaces. By "enough" we mean that any topological group is realized as the topological fundamental group $\pi_{1}^{\tau}\left(Z, z_{0}\right)$ of a locally wep-connected space $Z$ and for any space $X, \pi_{1}^{\tau}\left(X, x_{0}\right)$ may be approximated up to induced isomorphism on $\pi_{1}^{\tau}$ by a locally wep-connected space (Corollary 6.8). Thus for application of semicoverings to topological algebra, it suffices to consider locally wep-connected spaces.

The general structure of this paper is as follows. In section 2, we define what it means for a map to have continuous lifting of paths and homotopies and illustrate the importance of these properties to liftings of maps. Continuous lifting is then used to define the notion of semicovering in Section 3. Additionally, the properties of semicoverings are studied and contrasted with those of coverings in the classical sense. In section 4 , we use free topological groups to construct the fundamental Topgroupoid $\pi^{\tau} X$ of a space $X$ so that the underlying groupoid of $\pi^{\tau} X$ is the usual 
fundamental groupoid $\pi X$. Of utmost importance is the approximative characterization of $\pi^{\tau} X$ (4.7). This "topological approximation" is used in section 5 to show that a semicovering $p: Y \rightarrow X$ induces an open covering morphism $\pi^{\tau} p: \pi^{\tau} Y \rightarrow \pi^{\tau} X$ of Top-groupoids. In section 6 , we introduce and study locally wep-connected spaces, the spaces to which semicovering theory applies. Section 7 includes the classification of semicoverings and our main result: Theorem 7.1. The greatest difficulty in the classification, occurs in the construction of semicoverings and is treated separately in Section 7.1. The proof of Theorem 7.1 is completed in Section 7.2. Alternative statements of the classification in terms of enriched monodromy and continuous actions of topological groups on discrete spaces appear in Sections 7.3 and 7.4 respectively.

The author thanks Maria Basterra for helpful conversations and the referee for the suggestion of stating the classification of semicoverings as an equivalence of categories.

\section{Continuous lifting of paths and homotopies}

In general, sets of continuous functions $\operatorname{Top}(X, Y)$ of topological spaces will have the compact-open topology generated by subbasis sets $\langle K, U\rangle=\{f \mid f(K) \subseteq U\}$, $K \subseteq X$ compact, $U \subseteq Y$ open. Let $\mathcal{P} X$ denote the space of paths $\alpha: I=[0,1] \rightarrow X$ and $c_{x}$ denote the constant path at $x \in X$. If $\mathscr{B}$ is a basis for the topology of $X$ which is closed under finite intersection, sets of the form $\bigcap_{j=1}^{n}\left\langle K_{n}^{j}, U_{j}\right\rangle$ where $K_{n}^{j}=\left[\frac{j-1}{n}, \frac{j}{n}\right]$ and $U_{j} \in \mathscr{B}$ form a convenient basis for the topology of $\mathcal{P} X$.

For any fixed, closed subinterval $A \subseteq I$, let $T_{A}: I \rightarrow A$ be the unique, increasing, linear homeomorphism. For a path $\alpha \in \mathcal{P} X, \alpha_{A}=\left.\alpha\right|_{A} \circ T_{A}: I \rightarrow A \rightarrow X$ is the restricted path of $p$ to $A$. As a convention, if $A=\{t\} \subseteq I$, we let $\alpha_{A}=c_{\alpha(t)}$. Note that if $0=t_{0} \leqslant t_{1} \leqslant \ldots \leqslant t_{n}=1$, knowing the paths $\alpha_{\left[t_{i-1}, t_{i}\right]}$ for $i=1, \ldots, n$ uniquely determines $\alpha$. It is simple to describe concatenations of paths with this notation: If $\alpha_{1}, \alpha_{2}, \ldots, \alpha_{n} \in \mathcal{P} X$ such that $\alpha_{j}(1)=\alpha_{j+1}(0)$ for each $j=1, \ldots, n-1$, the $n$-fold concatenation of this sequence is the unique path $\beta=\alpha_{1} * \alpha_{2} * \cdots * \alpha_{n}$ such that $\beta_{K_{n}^{j}}=\alpha_{j}$ for each $j=1, \ldots, n$. It is an elementary fact of the compact-open topology that concatenation $\mathcal{P} X \times_{X} \mathcal{P} X=\{(\alpha, \beta) \mid \alpha(1)=\beta(0)\} \rightarrow \mathcal{P} X,(\alpha, \beta) \mapsto \alpha * \beta$ is continuous. If $\alpha \in \mathcal{P} X$, then $\alpha^{-1}(t)=\alpha(1-t)$ is the reverse of $\alpha$ and for a set $A \subseteq \mathcal{P} X, A^{-1}=\left\{\alpha^{-1} \mid \alpha \in A\right\}$. The operation $\alpha \mapsto \alpha^{-1}$ is a self-homeomorphism of $\mathcal{P} X$.

If $x, y \in X$, we take $(\mathcal{P} X)_{x}=\{\alpha \in \mathcal{P} X \mid \alpha(0)=x\},(\mathcal{P} X)^{y}=\{\alpha \in \mathcal{P} X \mid \alpha(1)=y\}$, and $\mathcal{P} X(x, y)=(\mathcal{P} X)_{x} \cap(\mathcal{P} X)^{y}$ to be subspaces of $\mathcal{P} X$. In notation, we do not distinguish a neighborhood from being an open set in $\mathcal{P} X$ or any of its subspaces but rather leave this to context. We also write $\Omega(X, x)$ for the space of loops $\mathcal{P} X(x, x)$ based at $x$. Each of these constructions gives either a functor Top $\rightarrow$ Top or Top $_{*} \rightarrow$ $\operatorname{Top}_{*}$. For instance, $(X, x) \mapsto\left((\mathcal{P} X)_{x}, c_{x}\right)$ is a functor which is $\mathcal{P} f(\alpha)=f \circ \alpha$ on morphisms.

Definition 2.1. A map $p: Y \rightarrow X$ has continuous lifting of paths if $\mathcal{P} p:(\mathcal{P} Y)_{y} \rightarrow$ $(\mathcal{P} X)_{p(y)}$ is a homeomorphism for each $y \in Y$.

Certainly every map with continuous lifting of paths has unique path lifting since unique path lifting is equivalent to the injectivity of each map $\mathcal{P} p$ in the above definition. Consequently, a map with continuous lifting of paths has the unique lifting 
property with respect to all path connected spaces [25, 2.2 Lemma 4]. The condition that $\mathcal{P} p$ be a homeomorphism is much stronger than the existence and uniqueness of lifts of paths since each inverse $L_{p}:(\mathcal{P} X)_{p(y)} \rightarrow(\mathcal{P} Y)_{y}$, which we refer to as the lifting homeomorphism, taking a path $\alpha$ to the unique lift $\tilde{\alpha}_{y}$ starting at $y$ is required to be continuous.

Let $\Delta_{2}=\left\{(s, t) \in I^{2} \mid s+t \leqslant 1\right\}$ be the 2 -simplex with edges $e_{1}, e_{2}, e_{3}$ opposite to vertices $(1,0),(0,1),(0,0)$ respectively. Let $\partial_{j}: e_{j} \hookrightarrow \Delta_{2}$ denote each inclusion and $(\Phi X)_{x}$ be the space of relative maps $\left(\Delta_{2}, e_{1}\right) \rightarrow(X,\{x\})$. If $\alpha, \beta \in(\mathcal{P} X)_{x}$ such that $\alpha(1)=\beta(1)$, then $\alpha \simeq \beta$ (rel. endpoints) if and only if there is a $\phi \in(\Phi X)_{x}$ such that $\alpha$ is $I \stackrel{\partial_{2}}{\longrightarrow} \Delta_{2} \stackrel{\phi}{\longrightarrow} X$ and $\beta$ is $I \stackrel{\cong}{\longrightarrow} e_{3} \stackrel{\partial_{3}}{\longrightarrow} \Delta_{2} \stackrel{\phi}{\longrightarrow} X$ (the first map is the inverse of the homeomorphic projection of $e_{3}$ onto $e_{2}=I$ ). Thus elements of $(\Phi X)_{x}$ are endpoint preserving homotopies of paths starting at $x$. Just as with path spaces, $\Phi: \mathbf{T o p}_{*} \rightarrow \mathbf{T o p}_{*}$ is a functor which is $\Phi f(\phi)=f \circ \phi$ on morphisms.

Definition 2.2. A map $p: Y \rightarrow X$ has continuous lifting of homotopies if

$$
\Phi p:(\Phi Y)_{y} \rightarrow(\Phi X)_{p(y)}
$$

is a homeomorphism for each $y \in Y$.

Let $\mathcal{G}$ be a (small) groupoid with source and target maps $s, t: \mathcal{G} \rightarrow O b(\mathcal{G})$. The star of $\mathcal{G}$ at $x \in O b(\mathcal{G})$ is the fiber $\mathcal{G}_{x}=\{g \in \mathcal{G} \mid s(g)=x\}$. Additionally, $\mathcal{G}^{y}=\{g \in$ $\mathcal{G} \mid t(g)=y\}, \mathcal{G}(x, y)=\mathcal{G}_{x} \cap \mathcal{G}^{y}$ and $\mathcal{G}(x)=\mathcal{G}(x, x)$. If $g \in \mathcal{G}(x, y)$, right and left multiplication by $g$ are $\rho_{g}: \mathcal{G}(w, x) \rightarrow \mathcal{G}(w, y), f \rightarrow f g$ and $\lambda_{g}: \mathcal{G}(y, z) \rightarrow \mathcal{G}(x, z), f \mapsto g f$ respectively.

Definition 2.3. A covering morphism is a functor $F: \mathcal{H} \rightarrow \mathcal{G}$ of groupoids such that $\mathcal{H}_{x} \rightarrow \mathcal{G}_{F(x)}, h \mapsto F(h)$ is a bijection for each $x \in O b(\mathcal{H})$. If $g \in \mathcal{G}_{F(x)}$, then $\tilde{g}_{x}$ denotes the unique $\tilde{g}_{x} \in \mathcal{H}_{x}$ such that $F\left(\tilde{g}_{x}\right)=g$. If $\mathcal{G}$ is a groupoid, $\operatorname{CovMor}(\mathcal{G})$ denotes the category of covering morphism $\mathcal{H} \rightarrow \mathcal{G}$. Morphisms in $\operatorname{CovMor}(\mathcal{G})$ are the obvious commuting triangles of functors.

Covering morphisms were first introduced as "regular morphism" by P.A. Smith [24] and are known to have rich application to group theory [18]. The next lemma follows directly from lifting arguments in classical covering space theory.

Lemma 2.4. Let $p: Y \rightarrow X$ be a map with continuous lifting of paths and homotopies.

1. Then $p$ induces a covering morphism $\pi p: \pi Y \rightarrow \pi X$ of fundamental groupoids.

2. If $p\left(y_{i}\right)=x_{i}, i=1,2$ and $\beta \in \mathcal{P} X\left(x_{1}, x_{2}\right)$, then $[\beta]$ lies in the image of

$$
\pi p: \pi Y\left(y_{1}, y_{2}\right) \rightarrow \pi X\left(x_{1}, x_{2}\right)
$$

if and only if $\tilde{\beta}_{y_{1}}(1)=y_{2}$.

3. The underlying set of $Y$ is a right $\pi X$-set in the sense that there are functions $p^{-1}\left(x_{1}\right) \times \pi X\left(x_{1}, x_{2}\right) \rightarrow p^{-1}\left(x_{2}\right),(y,[\alpha]) \mapsto y \cdot[\alpha]=\tilde{\alpha}_{y}(1)$ satisfying $(y \cdot[\alpha]) \cdot$ $[\beta]=y \cdot([\alpha * \beta])$ and $y \cdot\left[c_{x}\right]=y$.

To illustrate precisely why the notion of continuous lifting is worth considering in covering space theory and its generalizations, we generalize another well-known and useful lifting result $[\mathbf{6}, 10.5 .3]$. 
Lemma 2.5. Let $p: Y \rightarrow X$ be a map with continuous lifting of paths and homotopies and $W$ be a space whose path components are open and such that for each $w \in W$ evaluation $e v_{1}:(\mathcal{P} W)_{w} \rightarrow W, \beta \mapsto \beta(1)$ is quotient onto the path component of $w$. If $f: W \rightarrow X$ is any map such that $\pi f: \pi W \rightarrow \pi X$ lifts to a morphism $\Psi: \pi W \rightarrow \pi Y$ of groupoids (i.e., $\pi p \circ \Psi=\pi f$ ), then $\tilde{f}=O b(\Psi): W \rightarrow Y$ is continuous and $\Psi=\pi \tilde{f}$.

Proof. Since the path components of $W$ are open, it suffices to show the restriction of $\tilde{f}$ to each path component of $W$ is continuous. Thus it suffices to prove the lemma for $W$ path connected. Suppose $\tilde{f}\left(w_{0}\right)=y_{0}$ and $p\left(y_{0}\right)=x_{0}=f\left(w_{0}\right)$. Note that $\tilde{f}$ is determined as follows: given $w \in W$ and any path $\beta \in(\mathcal{P} W)_{w_{0}}$ such that $\alpha(1)=$ $w, \tilde{f}(w)=\widetilde{f \circ \alpha_{y_{0}}}(1)$. That this description of $\tilde{f}(w)$ does not depend on the choice of $w_{0}$ or $\beta \in(\mathcal{P} W)_{w_{0}}$ follows, in the usual manner, from the unique path lifting of $p$ and the assumption that $\Psi$ is a lift of $\pi f$. By functorality, $\mathcal{P} f:(\mathcal{P} W)_{w_{0}} \rightarrow$ $(\mathcal{P} X)_{x_{0}}$ is continuous and since $p$ has continuous lifting of paths, there is a lifting homeomorphism $L_{p}:(\mathcal{P} X)_{x_{0}} \rightarrow(\mathcal{P} Y)_{y_{0}}$. The diagram

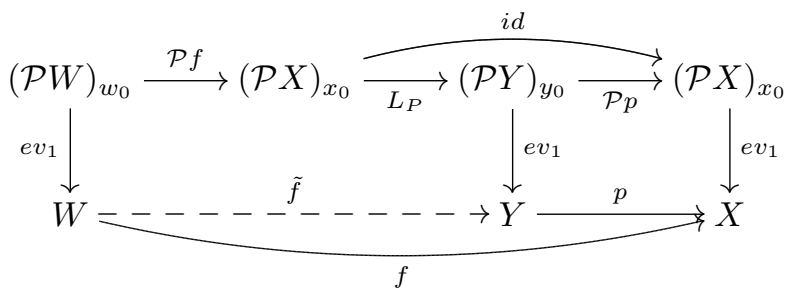

commutes and since the left-most vertical map is quotient, $\tilde{f}$ is continuous by the universal property of quotient spaces. Since $\pi p$ is a covering morphism and $\Psi$ and $\pi \tilde{f}$ are both lifts of $\pi f$, we have $\Psi=\pi \tilde{f}$.

A similar formulation is given in terms of the fundamental group $\pi_{1}$. We write $f_{*}: \pi_{1}\left(W, w_{0}\right) \rightarrow \pi_{1}\left(X, x_{0}\right)$ for the homomorphism induced by a map $f: W \rightarrow X$ which is $f\left(w_{0}\right)=x_{0}$ on basepoints.

Corollary 2.6. Let $p: Y \rightarrow X$ be a map with continuous lifting of paths and homotopies such that $p\left(y_{0}\right)=x_{0}$ and $W$ be a path connected space such that evaluation $e v_{1}:(\mathcal{P} W)_{w_{0}} \rightarrow W$ at 1 is quotient. A map $f: W \rightarrow X$ such that $f\left(w_{0}\right)=x_{0}$ has a unique lift $\tilde{f}: W \rightarrow Y$ such that $\tilde{f}\left(w_{0}\right)=y_{0}$ if and only if

$$
f_{*}\left(\pi_{1}\left(W, w_{0}\right)\right) \subseteq p_{*}\left(\pi_{1}\left(Y, y_{0}\right)\right) .
$$

\section{3. $\quad$ Semicovering maps}

Definition 3.1. A semicovering map is a local homeomorphism with continuous lifting of paths and homotopies.

When $p: Y \rightarrow X$ is a semicovering map, we refer to $Y$ as a semicovering space of $X$ and often refer to $p$ simply as a semicovering of $X$. If $p^{\prime}: Y^{\prime} \rightarrow X$ is another semicovering of $X$, a morphism of semicoverings is a map $f: Y \rightarrow Y^{\prime}$ such that the 
triangle

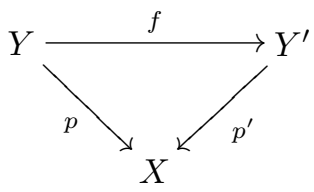

commutes. This defines a category $\mathbf{S C o v}(X)$ of semicoverings of $X$. Two semicoverings of $X$ are then equivalent if they are isomorphic in this category. A semicovering $p: Y \rightarrow X$ is connected if $Y$ is non-empty and path connected. Let $\operatorname{SCov}_{\mathbf{0}}(X)$ denote the full subcategory of connected semicoverings. A universal semicovering of $X$ is a semicovering initial in $\mathbf{S} \mathbf{C o v}_{\mathbf{0}}(X)$.

Remark 3.2. Every zero morphism $\emptyset \rightarrow X$ is vacuously a semicovering called the empty semicovering of $X$. The covering morphism induced by the empty semicovering is the unique covering morphism from the groupoid whose object set is empty to $\pi X$. Note that if $y \in Y$ and $x \in X$, any path $\alpha \in \mathcal{P} X(p(y), x)$ lifts to a path $\tilde{\alpha}_{y}$ such that $p\left(\tilde{\alpha}_{y}(1)\right)=x$. Thus if $X$ is path connected and $p: Y \rightarrow X$ is a semicovering map, then either $Y=\emptyset$ or $p$ is surjective.

Remark 3.3. It is evident from Lemma 2.5 that a semicovering has the homotopy lifting property with respect to locally path connected, simply connected spaces and is therefore a Serre fibration with discrete fibers.

It is well-known that if one does not restrict to spaces with universal coverings the composition of two connected covering maps is not always a covering map. On the other hand, it is straightforward from the definition of semicovering that the composition of two (not necessarily connected) semicoverings is a semicovering. In fact, connected semicoverings have the desirable "two out of three" property. The following lemma is an exercise in point-set topology.

Lemma 3.4. Let $p: X \rightarrow Y, q: Y \rightarrow Z$ and $r=q \circ p$ be surjective maps. If two of $p, q, r$ are local homeomorphisms, then so is the third. If two of $p, q, r$ have continuous lifting of paths and homotopies, then so does the third.

Corollary 3.5. Let $p: X \rightarrow Y, q: Y \rightarrow Z$, and $r=q \circ p$ be maps of path connected spaces. If two of $p, q, r$ are semicoverings, so is the third.

Proof. By Lemma 3.4 it suffices to show that if two of $p, q, r$ are semicoverings, the third map is surjective. Suppose $p\left(x_{0}\right)=y_{0}$ and $q\left(y_{0}\right)=z_{0}=r\left(x_{0}\right)$. If $p$ and $q$ are semicoverings, they are both surjective since $Y$ and $Z$ are path connected. Thus $r$ is surjective. If $q$ and $r$ are semicoverings and $y \in Y$ take $\alpha \in(\mathcal{P} Y)_{y_{0}}$ with $\alpha(1)=y$. Then $q \circ \alpha \in(\mathcal{P} Z)_{z_{0}}$ has unique lift $\widetilde{q \circ \alpha_{x_{0}}} \in(\mathcal{P} X)_{x_{0}}$ (with respect to $r$ ) with endpoint $x=\widetilde{q \circ \alpha_{x_{0}}}(1)$. Since $q \circ p \circ{\widetilde{q \circ \alpha_{x_{0}}}}=q \circ \alpha$ and $q$ has unique path lifting, we have $p \circ \widetilde{q \circ \alpha}_{x_{0}}=\alpha$. Therefore $p(x)=\alpha(1)=y$ and $p$ is surjective. Lastly, suppose $p$ and $r$ are semicoverings. Since $Z$ is path connected, $r$ is surjective and therefore $q$ is surjective.

To illustrate that the notion of semicovering actually generalizes the notion of covering, we check that every covering is a semicovering. 
Remark 3.6. If $p: Y \rightarrow X$ is a covering map and $U \subseteq X$ is an evenly covered neighborhood, then $p^{-1}(U)$ is the disjoint union $\amalg_{\lambda} V_{\lambda}$ of slices $V_{\lambda}$ over $U$ which are mapped homeomorphically onto $U$ by $p$. The collection of slices over evenly covered neighborhoods form a basis $\mathscr{B}_{p}$ for the topology of $Y$ which is closed under finite intersection. Consequently, the neighborhoods of the form $\bigcap_{j=1}^{n}\left\langle K_{n}^{j}, V_{j}\right\rangle, V_{j} \in \mathscr{B}_{p}$ give a basis for the topology of $\mathcal{P} Y$.

Proposition 3.7. For any space $X, \operatorname{Cov}(X)$ and $\operatorname{Cov}_{\mathbf{0}}(X)$ are full subcategories of $\operatorname{SCov}(X)$ and $\mathbf{S C o v}_{\mathbf{0}}(X)$ respectively.

Proof. Certainly, a covering map $p: Y \rightarrow X$ is a local homeomorphism. Suppose $p\left(y_{0}\right)=x_{0}$. Since covering maps uniquely lift paths and homotopies of paths,

$$
\mathcal{P} p:(\mathcal{P} Y)_{y_{0}} \rightarrow(\mathcal{P} X)_{x_{0}}
$$

and $\Phi p:(\Phi Y)_{y_{0}} \rightarrow(\Phi X)_{x_{0}}$ are bijective. Both are continuous by functorality. Let

$$
\mathcal{U}=\bigcap_{j=1}^{n}\left\langle K_{n}^{j}, U_{j}\right\rangle
$$

be a basic non-empty open neighborhood in $(\mathcal{P} Y)_{y_{0}}$, where each $U_{j} \in \mathscr{B}_{p}$. Since $\mathcal{U}$ is non-empty, there is a path $\tilde{\alpha}_{y_{0}} \in \mathcal{U}$ that is the lift of

$$
\alpha=p \circ \tilde{\alpha}_{y_{0}} \in \mathcal{V}=\left(\bigcap_{j=1}^{n}\left\langle K_{n}^{j}, p\left(U_{j}\right)\right\rangle\right) \cap\left(\bigcap_{j=1}^{n-1}\left\langle\left\{\frac{j}{n}\right\}, p\left(U_{j} \cap U_{j+1}\right)\right\rangle\right) \subseteq(\mathcal{P} X)_{x_{0}} .
$$

Clearly $\mathcal{P} p(\mathcal{U}) \subseteq \mathcal{V}$. The lift $\tilde{\alpha}_{y_{0}}$ has the following description: There are homeomorphisms $h_{j}: p\left(U_{j}\right) \rightarrow U_{j}$ such that $p \circ h_{j}$ is the identity of $p\left(U_{j}\right)$. For each $t \in K_{n}^{j}$, we have $\tilde{\alpha}_{y_{0}}(t)=h_{j} \circ \alpha(t)$. Note that if $\beta$ is any other path in $\mathcal{V}$, the unique lift $\tilde{\beta}_{y_{0}} \in(\mathcal{P} Y)_{y_{0}}$ is defined in the same way, that is, for each $t \in K_{n}^{j}, \tilde{\beta}_{y_{0}}(t)=h_{j} \circ \beta(t)$. The equality $\mathcal{P} p(\mathcal{U})=\mathcal{V}$ implies that $\mathcal{P} p$ is open. A completely analogous argument may be used to show that $\Phi p:(\Phi Y)_{y_{0}} \rightarrow(\Phi X)_{x_{0}}$ is a homeomorphism. One may proceed by viewing $\Delta_{2}$ as a simplicial complex and taking a basic open neighborhood of a homotopy $\tilde{G}_{y_{0}} \in(\Phi Y)_{y_{0}}$ to be of the form $\mathcal{U}=\bigcap_{\sigma \in s d_{n}\left(\Delta_{2}\right)}\left\langle\sigma, U_{\sigma}\right\rangle$ where the intersection is taken over 2-simplices $\sigma$ in the n-th barycentric subdivision $s d_{n}\left(\Delta_{2}\right)$ of $\Delta_{2}$ and $U_{\sigma} \in \mathscr{B}_{p}$. Then

$$
\mathcal{V}=\left(\bigcap_{\sigma \in s d_{n}\left(\Delta_{2}\right)}\left\langle\sigma, p\left(U_{\sigma}\right)\right\rangle\right) \cap\left(\bigcap_{e=\sigma \cap \sigma^{\prime}}\left\langle e, p\left(U_{\sigma} \cap U_{\sigma^{\prime}}\right)\right\rangle\right)
$$

is an open neighborhood of $G=p \circ \tilde{G}_{y_{0}}$ satisfying $\Phi p(\mathcal{U})=\mathcal{V}$. Here the second intersection ranges over all 1-simplices which are the intersection of two 2-simplices in $s d_{n}\left(\Delta_{2}\right)$. Thus every covering is a semicovering and $\mathbf{C o v}(X)$ and $\mathbf{C o v}_{\mathbf{0}}(X)$ are full subcategories.

Example 3.8. Since every covering is a semicovering, the composition of covering maps is always a semicovering map even if it is not a covering map. This fact alone provides simple examples of semicoverings which are not coverings. In the non-connected case, 
one can check that $S^{1} \times\{1,2, \ldots\} \rightarrow S^{1},(z, n) \rightarrow z^{n}$ is a semicovering but not a covering. There are connected semicoverings of the Hawaiian earring

$$
\mathbb{H} \mathbb{E}=\bigcup_{n \geqslant 1}\left\{(x, y) \in \mathbb{R}^{2} \mid\left(x-\frac{1}{n}\right)^{2}+y^{2}=\frac{1}{n^{2}}\right\}
$$

which are not coverings; variations of the semicovering space which is the answer to Exercise 6 in Chapter 1.3 of Hatcher [15] illustrate the extensiveness of semicoverings of non-semilocally 1-connected spaces beyond coverings.

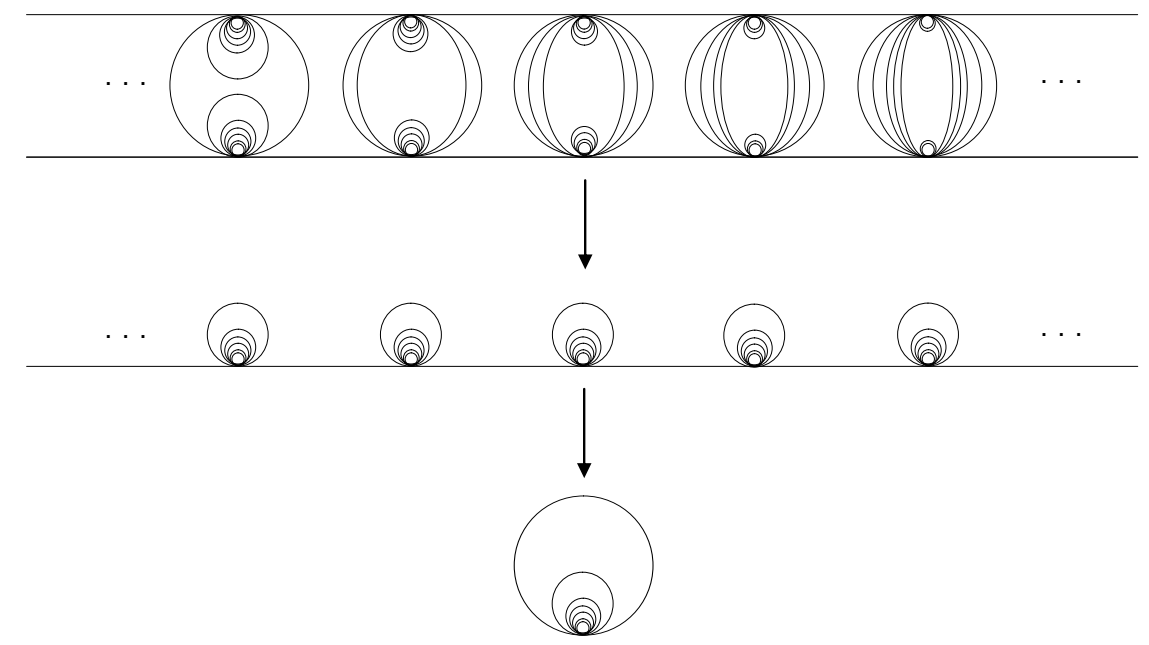

Figure 1: The bottom map is a covering of $\mathbb{H} \mathbb{E}$ with infinite fiber $\mathbb{Z}$. The top map is a two-sheeted covering of the covering, however, the composition fails to be a covering map since it fails local triviality. Thus the composition is a semicovering of $\mathbb{H} \mathbb{E}$ which is not a covering.

Remark 3.9. Recall that if $p: Y \rightarrow X$ is a covering map, $U$ is an evenly covered neighborhood of $x$ in $X$, and $i: U \rightarrow X$ is the inclusion, then

$$
i_{*}\left(\pi_{1}(U, x)\right) \subseteq \bigcap_{y \in p^{-1}(x)} p_{*}\left(\pi_{1}(Y, y)\right)
$$

in $\pi_{1}(X, x)$. Since a semicovering need not satisfy local triviality, one can only expect a space $X$ to inherit a weak version of this property given the existence of a semicovering $p: Y \rightarrow X$ : If $p: Y \rightarrow X$ is a semicovering, $x \in X$, and $y \in p^{-1}(x)$, there is an open neighborhood $V$ of $y$ mapped homeomorphically onto $U=p(V)$. This gives the inclusion $i_{*}\left(\pi_{1}(U, x)\right) \subseteq p_{*}\left(\pi_{1}(Y, y)\right)$ only for the single point $y$ in the fiber $p^{-1}(x)$. Indeed, for a semicovering which is not a covering, like the one in the above example, and any neighborhood $U$ of $x$, the inclusion $i_{*}\left(\pi_{1}(U, x)\right) \subseteq p_{*}\left(\pi_{1}(Y, y)\right)$ may only hold for finitely many $y$ in the fiber $p^{-1}(x)$.

It is also worthwhile to note that semicoverings admit an adequate theory of pullbacks. 
Proposition 3.10. If $p: Y \rightarrow X$ is a semicovering of $X, f: W \rightarrow X$ is a map, and $W \times_{X} Y=\{(w, y) \mid f(w)=p(y)\}$ is the pullback, the projection $f^{*} p: W \times_{X} Y \rightarrow W$ is a semicovering of $W$. Moreover, $f^{*}: \mathbf{S C o v}(X) \rightarrow \mathbf{S C o v}(W)$ is a functor

Proof. If $(w, y) \in W \times_{X} Y$, let $U$ be an open neighborhood of $y$ in $Y$ which is mapped homeomorphically onto $p(U)$ and $V=f^{-1}(p(U)) \subseteq W$. Now $(V \times U) \cap W \times_{X} Y$ is an open neighborhood of $(w, y)$ in $W \times_{X} Y$ mapped homeomorphically onto $V$ by $f^{*} p$. Let $q: W \times_{X} Y \rightarrow Y$ be the projection onto the second coordinate and fix $\left(w_{0}, y_{0}\right) \in$ $W \times_{X} Y$ so that $f\left(w_{0}\right)=x_{0}=p\left(y_{0}\right)$. Consider the pullback square

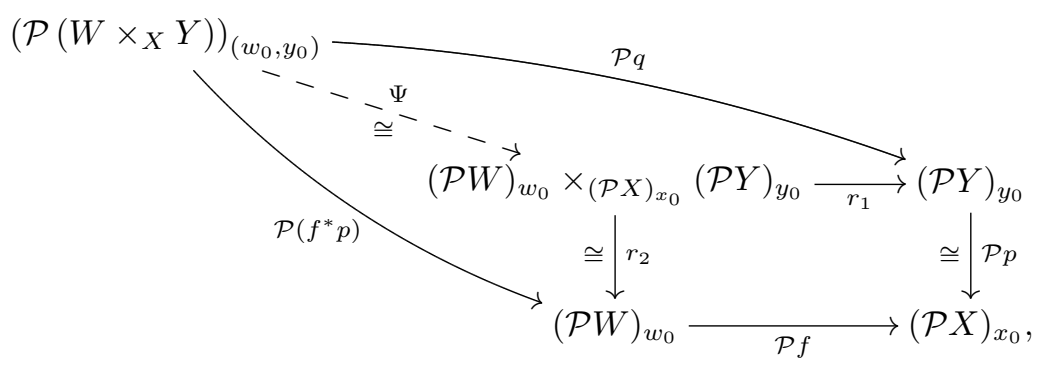

where $r_{1}, r_{2}$ are the projections. The maps $\mathcal{P}\left(f^{*} p\right)$ and $\mathcal{P} q$ induce the canonical homeomorphism $\Psi$. Since $\mathcal{P} p$ is a homeomorphism by assumption, categorical considerations give that $r_{2}$ is a homeomorphism. Thus $\mathcal{P}\left(f^{*} p\right)$ is a homeomorphism. The same argument may be used to show that $\Phi\left(f^{*} p\right):\left(\Phi\left(W \times_{X} Y\right)\right)_{\left(w_{0}, x_{0}\right)} \rightarrow(\Phi W)_{w_{0}}$ is a homeomorphism.

The fact that a morphism $g: Y \rightarrow Y^{\prime}$ of semicoverings $p$ and $p^{\prime}$ of $X$ induces a map $f^{*} g: W \times_{X} Y \rightarrow W \times_{X} Y^{\prime}$ such that $f^{*} p^{\prime} \circ f^{*} g=f^{*} p$ (i.e., a morphism $f^{*} p \rightarrow f^{*} p^{\prime}$ ) follows from the universal property of $W \times{ }_{X} Y^{\prime}$. Thus $f^{*}: \operatorname{SCov}(X) \rightarrow \operatorname{SCov}(W)$ is a functor.

Proposition 3.11. If $p: Y \rightarrow X$ and $f: Z \rightarrow X$ are connected semicoverings and $p$ is universal, the induced semicovering $f^{*} p$ of $Z$ is universal.

\section{Top-groupoids and $\pi^{\tau} X$}

In this section, we present the construction of a topologically enriched fundamental groupoid $\pi^{\tau}$ to play a prominent role in the classification of semicoverings. We mean enrichment over Top in the sense of [20].

A Top-category is a category $\mathcal{C}$ enriched over Top, the category of topological spaces, in the sense that each hom-set is equipped with a topology such that all composition maps are continuous. For instance, we view Set as a Top-category by viewing each set as a discrete space and giving sets of functions the topology of point-wise convergence. Given Top-categories $\mathcal{A}$ and $\mathcal{B}$, a Top-functor is a functor $F: \mathcal{A} \rightarrow \mathcal{B}$ such that each function $\mathcal{A}\left(a_{1}, a_{2}\right) \rightarrow \mathcal{B}\left(F\left(a_{1}\right), F\left(a_{2}\right)\right)$ is continuous. A Top-natural transformation of Top-functors is a natural transformation of the underlying functors. The category of Top-functors $\mathcal{A} \rightarrow \mathcal{B}$ and Top-natural transformations is denoted TopFunc $(\mathcal{A}, \mathcal{B})$. 
When the underlying category of a Top-category $\mathcal{C}$ is a groupoid and each inversion map $\mathcal{C}(a, b) \rightarrow \mathcal{C}(b, a)$ is continuous, we call $\mathcal{C}$ a Top-groupoid. The category of Topgroupoids will be denoted TopGrpd. The author emphasizes that the notion of Top-groupoid is distinct from that of topological groupoid which refers to a groupoid internal to Top.

To construct $\pi^{\tau}$ we pass through two topologized versions of the fundamental group: The path component space $\pi_{0}^{\text {qtop }} Z$ of a space $Z$ is the set of path components $\pi_{0} Z$ viewed canonically as a quotient space of $Z$. That $\pi_{0}^{q t o p}$ gives endofunctors of Top and Top $_{*}$ follows directly from the universal property of quotient spaces. The group $\pi_{1}^{\text {qtop }}(X, x)=\pi_{0}^{q t o p} \Omega(X, x)$ is the quasitopological fundamental group of $(X, x)$ and is characterized by the canonical map $h: \Omega(X, x) \rightarrow \pi_{1}^{q t o p}(X, x)$ identifying homotopy classes of loops being quotient. This group is "quasitopological" in the sense that inversion is continuous and left and right translations by fixed elements are continuous. It is known that $\pi_{1}^{q t o p}$ is a homotopy invariant which takes values in the category qTopGrp of quasitopological groups and continuous group homomorphisms [9], however, $\pi_{1}^{q t o p}(X, x)$ often fails to be a topological group $[\mathbf{3}, \mathbf{1 0}, \mathbf{1 1}]$.

In [4], this failure is repaired within qTopGrp by noticing that the forgetful functor TopGrp $\rightarrow$ qTopGrp has a left adjoint $\tau$ such that the two triangles in

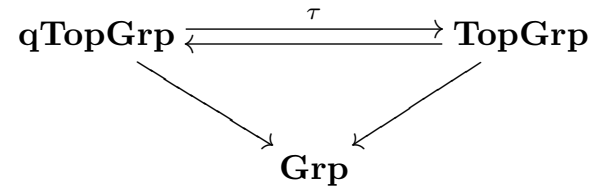

commute (the unlabeled arrows are forgetful functors). To construct $\tau$ explicitly, let $F_{M}(G)$ be the free (Markov) topological group on the underlying space of $G$ and $m_{G}: F_{M}(G) \rightarrow G$ be the multiplication of letters induced by the identity of $G$. Let $\tau(G)$ be the underlying group of $G$ with the quotient topology with respect to $m_{G}$. Since $F_{M}(G)$ is a topological group, so is the quotient group $\tau(G)$. Applying $\tau$ to a quasitopological group $G$ has the effect of removing the fewest number of open sets from the topology of $G$ so that one obtains a topological group. Thus $\tau(G)=G$ if and only if $G$ is already a topological group. It then makes sense to define $\pi_{1}^{\tau}=\tau \circ \pi_{1}^{q t o p}:$ Top $_{*} \rightarrow$ TopGrp.

Proposition 4.1. The topology of $\pi_{1}^{\tau}\left(X, x_{0}\right)$ is the finest group topology on $\pi_{1}\left(X, x_{0}\right)$ such that $h: \Omega\left(X, x_{0}\right) \rightarrow \pi_{1}\left(X, x_{0}\right)$ is continuous.

Proof. Suppose $\pi_{1}\left(X, x_{0}\right)$ is endowed with a topology making it a topological group and such that $\Omega\left(X, x_{0}\right) \rightarrow \pi_{1}\left(X, x_{0}\right)$ is continuous. The identity

$$
\pi_{1}^{q t o p}\left(X, x_{0}\right) \rightarrow \pi_{1}\left(X, x_{0}\right)
$$

is continuous by the universal property of quotient spaces and since $\pi_{1}\left(X, x_{0}\right)$ is a topological group, the adjoint is the continuous identity

$$
\pi_{1}^{\tau}\left(X, x_{0}\right)=\tau\left(\pi_{1}^{q t o p}\left(X, x_{0}\right)\right) \rightarrow \pi_{1}\left(X, x_{0}\right) .
$$

Thus the topology of $\pi_{1}^{\tau}\left(X, x_{0}\right)$ is finer than that of $\pi_{1}\left(X, x_{0}\right)$.

The above constructions are now extended from groups to groupoids. 
Definition 4.2. A qTop-groupoid is a (small) groupoid $\mathcal{G}$ where the hom-sets $\mathcal{G}(x, y)$ are equipped with topologies such that each multiplication $\mathcal{G}(x, y) \times \mathcal{G}(y, z) \rightarrow \mathcal{G}(x, z)$ is separately continuous (i.e., continuous in each variable) and each inversion function $\mathcal{G}(x, y) \rightarrow \mathcal{G}(y, x)$ is continuous. A morphism of qTop-groupoids is a functor $F: \mathcal{G} \rightarrow \mathcal{G}^{\prime}$ such that each function $F: \mathcal{G}(x, y) \rightarrow \mathcal{G}^{\prime}(F(x), F(y)), f \mapsto F(f)$ is continuous. Let qTopGrpd be the category of qTop-groupoids.

Note that $\mathcal{G}(x, y) \times \mathcal{G}(y, z) \rightarrow \mathcal{G}(x, z)$ is continuous in each variable if and only if all translations $\lambda_{g}: \mathcal{G}(w, x) \rightarrow \mathcal{G}(w, y)$ and $\rho_{g}: \mathcal{G}(y, z) \rightarrow \mathcal{G}(x, z)$ are homeomorphisms. Since every Top-groupoid is a qTop-groupoid, TopGrpd is a full subcategory of qTopGrpd.

Remark 4.3. The separate continuity of multiplication in quasitopological groups and qTop-groupoids can be captured using a certain cartesian closed monoidal structure on the category of spaces: See [8, Remark 1.15]. This topology is sometimes called the cross topology [16]. Let $X \otimes Y$ be the set-theoretic product with the weak topology with respect to the projection fibers $X \times\{y\},\{x\} \times Y$. A map $X \times Y \rightarrow Z$ on the usual product is separately continuous if and only if $X \otimes Y \rightarrow Z$ is continuous.

Proposition 4.4. Let $\pi^{q t o p} X$ denote the fundamental groupoid of $X$, where $\pi^{q t o p} X\left(x_{1}, x_{2}\right)$ is viewed as the quotient space $\pi_{0}^{q t o p}\left(\mathcal{P} X\left(x_{1}, x_{2}\right)\right)$. This gives the fundamental groupoid the structure of a qTop-groupoid. Moreover, $\pi^{q t o p}$ : Top $\rightarrow$ qTopGrpd is a functor.

Proof. By applying $\pi_{0}^{q t o p}$ to operations of left concatenation $\alpha \rightarrow \alpha * \beta$, right concatenation $\alpha \rightarrow \beta * \alpha$, and inversion $\alpha \mapsto \alpha^{-1}$ on path spaces, one observes that $\pi^{q t o p} X$ is indeed a qTop-groupoid. Similarly, a map $f: X \rightarrow Y$ induces the map $\pi_{0}^{t o p}(\mathcal{P}(f)): \pi^{q t o p} X\left(x_{1}, x_{2}\right) \rightarrow \pi^{q t o p} Y\left(f\left(x_{1}\right), f\left(x_{2}\right)\right),[\alpha] \mapsto[f \circ \alpha]$.

The following lemma extends the definition of $\tau$ from groups to groupoids by applying the group-valued $\tau$ to vertex groups and extending via translations. It is reminiscent of the use of the adjoint functor theorem in $[7]$ to construct colimits of topological groupoids.

Lemma 4.5. The forgetful functor TopGrpd $\rightarrow$ qTopGrpd has a left adjoint $\tau:$ qTopGrpd $\rightarrow$ TopGrpd which is the identity on underlying groupoids.

Proof. Let $\mathcal{G}$ be a qTop-groupoid. For each $x \in O b(\mathcal{G})$, let $\tau(\mathcal{G})(x)$ be the topological group $\tau(\mathcal{G}(x))$. If $x \neq y$ and $\mathcal{G}(x, y) \neq \emptyset$, let $\tau(\mathcal{G})(x, y)$ have the topology generated by the sets $U g=\{u g \mid u \in U\}$ where $g \in \mathcal{G}(x, y)$ and $U$ is open in $\tau(\mathcal{G})(x)$. Since $U g_{1} g_{2}^{-1}$ is open in $\tau(\mathcal{G})(x)$ for all $g_{1}, g_{2} \in \mathcal{G}(x, y)$, the right translations $\rho_{g}: \tau(\mathcal{G})(x) \rightarrow$ $\tau(\mathcal{G})(x, y)$ are homeomorphisms. Note that if $g \in \mathcal{G}(x, y)$, then $\lambda_{g^{-1}} \circ \rho_{g}: \mathcal{G}(x) \rightarrow$ $\mathcal{G}(y), h \mapsto g^{-1} h g$ is an isomorphism of quasitopological groups. The functorality of $\tau:$ qTopGrp $\rightarrow$ TopGrp then gives that the same homomorphism $\tau(\mathcal{G})(x) \rightarrow$ $\tau(\mathcal{G})(y)$ is an isomorphism of topological groups. Thus all left translations $\lambda_{g}: \tau(\mathcal{G})(y) \rightarrow \tau(\mathcal{G})(x, y)$ are homeomorphisms. It is now straightforward to use the fact that the vertex groups $\tau(\mathcal{G})(x)$ are topological groups to see that $\tau(\mathcal{G})$ is a Topgroupoid. A morphism $F: \mathcal{G} \rightarrow \mathcal{G}^{\prime}$ of qTop-groupoids induces a morphism $\tau(F): \tau(\mathcal{G}) \rightarrow \tau\left(\mathcal{G}^{\prime}\right)$ of Top-groupoids since the group-valued $\tau$ on the vertex groups 
gives continuous homomorphisms $\tau(\mathcal{G})(x) \rightarrow \tau\left(\mathcal{G}^{\prime}\right)(F(x))$ and continuity extends to all hom-sets via translations. We use a similar argument to illustrate the universal property of $\tau(\mathcal{G})$. Suppose $\mathcal{G}^{\prime}$ is a Top-groupoid and $F: \mathcal{G} \rightarrow \mathcal{G}^{\prime}$ is a morphism of qTop-groupoids. It suffices to show that each function

$$
F: \tau(\mathcal{G})(x, y) \rightarrow \mathcal{G}^{\prime}(F(x), F(y))
$$

is continuous. Note that for each $x \in O b(\mathcal{G}), F: \mathcal{G}(x) \rightarrow \mathcal{G}^{\prime}(F(x))$ is a continuous group homomorphism from a quasitopological group to a topological group. The adjoint homomorphism $F: \tau(\mathcal{G})(x)=\tau(G(x)) \rightarrow \mathcal{G}^{\prime}(F(x))$ is also continuous. Again, extend via translations to find that $F: \tau(\mathcal{G})(x, y) \rightarrow \mathcal{G}^{\prime}(F(x), F(y))$ is continuous in general.

By construction, the vertex groups $\tau(\mathcal{G})(x)$ of $\tau(\mathcal{G})$ are the topological groups $\tau(\mathcal{G}(x))$. Since each identity $\mathcal{G}(x) \rightarrow \tau(\mathcal{G})(x)$ is continuous, it follows that the identity functor $\mathcal{G} \rightarrow \tau(\mathcal{G})$ is a morphism of qTop-groupoids. Similar to the situation with groups, a qTop-groupoid $\mathcal{G}$ is a Top-groupoid if and only if $\mathcal{G}=\tau(\mathcal{G})$.

Definition 4.6. The fundamental Top-groupoid of a topological space $X$ is the Topgroupoid $\pi^{\tau} X=\tau\left(\pi^{q t o p} X\right)$.

For practical purposes, we provide an alternative construction of $\pi^{\tau} X$. The following approximation technique, which is possible since the existence of $\pi^{\tau} X$ is already known, extends to groupoids the well-known process of inductively forming quotient topologies on groups. The group case of what is given here is laid out in more detail in $[4]$.

Approximation of $\tau(\mathcal{G})$ 4.7. Let $\mathcal{G}=\mathcal{G}_{0}$ be a qTop-groupoid. Construct qTopgroupoids $\mathcal{G}_{\zeta}$ inductively so that if $\zeta$ is a successor ordinal, the topology of $\mathcal{G}_{\zeta}(x, y)$ (provided it is non-empty) is the quotient topology with respect to the sum of multiplication maps

$$
\mu: \coprod_{a \in O b(\mathcal{G})} \mathcal{G}_{\zeta-1}(x, a) \times \mathcal{G}_{\zeta-1}(a, y) \rightarrow \mathcal{G}_{\zeta}(x, y) .
$$

If $\zeta$ is a limit ordinal, the topology of $\mathcal{G}_{\zeta}(x, y)$ is the intersection of the topologies of $\mathcal{G}_{\eta}(x, y)$ for $\eta<\zeta$.

Lemma 4.8. Let $\mathcal{G}$ be a qTop-groupoid.

1. $\mathcal{G}_{\zeta}$ is a qTop-groupoid for each $\zeta$.

2. The identities $\mathcal{G} \rightarrow \mathcal{G}_{\zeta} \rightarrow \mathcal{G}_{\zeta+1} \rightarrow \tau(\mathcal{G})$ are morphisms of qTop-groupoids for each $\zeta$.

3. $\tau\left(\mathcal{G}_{\zeta}\right)=\tau(\mathcal{G})$ for each $\zeta$.

4. $\mathcal{G}_{\zeta}$ is a Top-groupoid if and only if $\mathcal{G}_{\zeta}=\tau(\mathcal{G})$ if and only if $\mathcal{G}_{\zeta}(x, y)=\mathcal{G}_{\zeta+1}(x, y)$ for all $x, y \in O b(\mathcal{G})$.

5. There is an ordinal number $\zeta_{0}$ such that $\mathcal{G}_{\zeta}=\tau(\mathcal{G})$ for each $\zeta \geqslant \zeta_{0}$.

Proof. 1. The continuity of translations and inversion in $\mathcal{G}_{\zeta}$ follows from a straightforward transfinite induction argument. When $\zeta$ is a successor, continuity follows in 
each case from the universal property of one of the quotient maps $\mu$. The case when $\zeta$ is a limit ordinal is even clearer.

2 . Procede by transfinite induction. For successor ordinal $\zeta$, each map $\mathcal{G}_{\zeta-1}(x, y) \rightarrow$ $\mathcal{G}_{\zeta}(x, y)$ is continuous since

$$
\mathcal{G}_{\zeta-1}(x, y) \times\left\{i d_{y}\right\} \subset \coprod_{a \in O b(G)} \mathcal{G}_{\zeta-1}(x, a) \times \mathcal{G}_{\zeta-1}(a, y)
$$

and $\mu$ is continuous. Thus if $\mathcal{G} \rightarrow \mathcal{G}_{\zeta-1}$ is a morphism of qTop-groupoids, so is $\mathcal{G} \rightarrow \mathcal{G}_{\zeta}$. Additionally, the left vertical map in the following diagram is quotient.

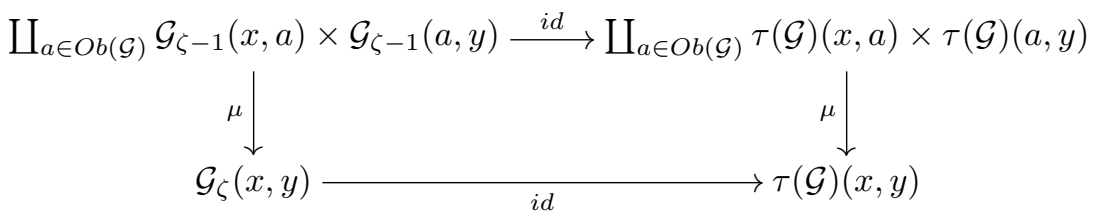

Therefore, if the top map is continuous, so is the bottom map. The case for limit ordinals is clear.

3. Since $i d: \mathcal{G}_{\zeta} \rightarrow \tau(\mathcal{G})$ is a morphism of qTop-groupoids so is $i d: \tau\left(\mathcal{G}_{\zeta}\right) \rightarrow$ $\tau(\tau(\mathcal{G}))=\tau(\mathcal{G})$. By 1., id: $\mathcal{G} \rightarrow \tau\left(\mathcal{G}_{\zeta}\right)$ is a morphism of qTop-groupoids whose adjoint is the inverse $i d: \tau(\mathcal{G}) \rightarrow \tau\left(\mathcal{G}_{\zeta}\right)$.

4. The first biconditional is clear from 3 . The second is clear from the observation that $\mathcal{G}_{\zeta}$ is a Top-groupoid if and only if $\mu: \coprod_{a \in O b(\mathcal{G})} \mathcal{G}_{\zeta}(x, a) \times \mathcal{G}_{\zeta}(a, y) \rightarrow \mathcal{G}_{\zeta}(x, y)$ is continuous for each $x, y \in O b(\mathcal{G})$.

5. For each ordinal $\zeta$, let $A_{\zeta}=\coprod_{x, y \in O b(\mathcal{G})} \mathcal{G}_{\zeta}(x, y)$ be the disjoint union of homspaces and $\mathscr{T}_{\zeta}$ be the topology of $A_{\zeta}$. 1. gives that $\mathscr{T}_{\zeta+1} \subseteq \mathscr{T}_{\zeta} \subseteq \mathscr{T}_{0}$ for each $\zeta$ and 4 . implies that $\mathscr{T}_{\zeta+1}=\mathscr{T}_{\zeta}$ if and only if $\mathcal{G}_{\zeta}=\tau(\mathcal{G})$. Suppose $\mathcal{G}_{\zeta} \neq \tau(\mathcal{G})$ for each $\zeta$. Thus $\mathscr{T}_{\zeta}-\mathscr{T}_{\zeta+1} \neq \emptyset$ for each ordinal $\zeta$, contradicting the fact there is no injection of ordinal numbers into the power set of $\mathscr{T}_{0}$. Thus there is an ordinal $\zeta_{0}$ such that $\mathcal{G}_{\zeta_{0}}=\tau(\mathcal{G})$. Since $\mathcal{G}_{\zeta_{0}} \rightarrow \mathcal{G}_{\zeta} \rightarrow \tau(\mathcal{G})$ are morphisms of $\mathbf{q T o p}$-groupoids whenever $\zeta \geqslant \zeta_{0}$, it follows that $\mathcal{G}_{\zeta}=\tau(\mathcal{G})$ for all $\zeta \geqslant \zeta_{0}$.

Corollary 4.9. For each $x_{1}, x_{2} \in X$, the canonical maps

$$
h: \mathcal{P} X\left(x_{1}, x_{2}\right) \rightarrow \pi^{\tau} X\left(x_{1}, x_{2}\right)
$$

identifying homotopy classes of paths are continuous.

Proof. The topology of $\pi^{\tau} X\left(x_{1}, x_{2}\right)$ is coarser than that of $\pi^{q t o p} X\left(x_{1}, x_{2}\right)$ and $h: \mathcal{P} X\left(x_{1}, x_{2}\right) \rightarrow \pi^{q t o p} X\left(x_{1}, x_{2}\right)$ is continuous by definition.

\section{Open covering morphisms}

The main purpose of this section is to use the inductive description of $\pi^{\tau} X=$ $\tau\left(\pi^{q t o p} X\right)$ to show that a semicovering induces an open covering morphism on Topfundamental groupoids in the following sense.

Definition 5.1. A Top-functor $F: \mathcal{A} \rightarrow \mathcal{B}$ is open if each map $F: \mathcal{A}\left(a_{1}, a_{2}\right) \rightarrow$ $\mathcal{B}\left(F\left(a_{1}\right), F\left(a_{2}\right)\right)$ is an open map. 
Thus an open covering morphism $F: \mathcal{H} \rightarrow \mathcal{G}$ of Top-groupoids is a covering morphism such that each map $F: \mathcal{H}\left(a_{1}, a_{2}\right) \rightarrow \mathcal{G}\left(F\left(a_{1}\right), F\left(a_{2}\right)\right)$ is an open embedding. If $\mathcal{G}$ is a Top-groupoid, OCovMor $(\mathcal{G})$ denotes the category of open covering morphisms $\mathcal{H} \rightarrow \mathcal{G}$. A morphism of open covering morphisms $p: \mathcal{H} \rightarrow \mathcal{G}$ and $p^{\prime}: \mathcal{H}^{\prime} \rightarrow \mathcal{G}$ is a Top-functor $F: \mathcal{H} \rightarrow \mathcal{H}^{\prime}$ such that $p^{\prime} \circ F=p$. The following proposition will be used in our classification of semicoverings and implies that every morphism of open covering morphism is an open covering morphism itself.

Proposition 5.2. Suppose $\mathcal{A}, \mathcal{B}, \mathcal{C}$ are Top-groupoids and $p: \mathcal{A} \rightarrow \mathcal{B}, q: \mathcal{B} \rightarrow \mathcal{C}$, and $r=q \circ p$ are functors.

1. If $p$ and $q$ are open covering morphisms, then so is $r$.

2. If $q$ and $r$ are open covering morphisms, then so is $p$.

3. If $p$ and $r$ are open covering morphisms and $O b(p)$ is surjective, then $q$ is an open covering morphism.

Proof. If we remove each appearance of "open" in the statement of the proposition, we obtain a known property of covering morphisms: see [6, 10.2.3]. Thus, for 1.-3. it suffices to check that the functor in question is an open Top-functor. This is elementary and is left to the reader.

Remark 5.3. One may construct pullbacks of Top-groupoids in the obvious way and extend the statements 10.7.2-10.7.5 in [6] to be stated in terms of semicoverings and open covering morphisms. For instance, if $\mathcal{A}, \mathcal{B}, \mathcal{G}$ are Top-groupoids, $p: \mathcal{A} \rightarrow \mathcal{G}$ is an open covering morphism and $F: \mathcal{B} \rightarrow \mathcal{G}$ is a Top-functor, the projection $F^{*} p: \mathcal{A} \times{ }_{\mathcal{G}} \mathcal{B} \rightarrow \mathcal{B}$ of Top-groupoids is an open covering morphism.

Lemma 5.4. If $p: Y \rightarrow X$ is a semicovering such that $p\left(y_{i}\right)=x_{i}, i=1,2$, the map $\mathcal{P} p: \mathcal{P} Y\left(y_{1}, y_{2}\right) \rightarrow \mathcal{P} X\left(x_{1}, x_{2}\right)$ is an open embedding.

Proof. Since $\mathcal{P} p: \mathcal{P} Y\left(y_{1}, y_{2}\right) \rightarrow \mathcal{P} X\left(x_{1}, x_{2}\right)$ is the restriction of the homeomorphism $\mathcal{P} p:(\mathcal{P} Y)_{y_{1}} \rightarrow(\mathcal{P} X)_{x_{1}}$, it suffices to show the image of $\mathcal{P} p$ is open in $\mathcal{P} X\left(x_{1}, x_{2}\right)$. Let $\alpha \in \mathcal{P} X\left(x_{1}, x_{2}\right)$ such that $\tilde{\alpha}_{y_{1}} \in \mathcal{P} Y\left(y_{1}, y_{2}\right)$. Let $\mathcal{U}=\bigcap_{j=1}^{n}\left\langle K_{n}^{j}, U_{j}\right\rangle$ be an open neighborhood of $\tilde{\alpha}_{y_{1}}$ in $(\mathcal{P} Y)_{y_{1}}$ such that $\left.p\right|_{U_{n}}: U_{n} \rightarrow p\left(U_{n}\right)$ is a homeomorphism. Since $\mathcal{P} p:(\mathcal{P} Y)_{y_{1}} \cong(\mathcal{P} X)_{x_{1}}, \mathcal{W}=\mathcal{P} p(\mathcal{U}) \cap \mathcal{P} X\left(x_{1}, x_{2}\right)$ is an open neighborhood of $\alpha$ in $\mathcal{P} X\left(x_{1}, x_{2}\right)$. If $\beta \in \mathcal{W}$, then $\mathcal{U}$ is an open neighborhood of $\tilde{\beta}_{y_{1}}$ in $(\mathcal{P} Y)_{y_{1}}$. In particular, $\tilde{\beta}_{y_{1}}(1) \in U_{n} \cap p^{-1}\left(x_{2}\right)=\left\{y_{2}\right\}$. Therefore $\tilde{\beta}_{y_{1}} \in \mathcal{P} Y\left(y_{1}, y_{2}\right)$ giving the inclusion $\mathcal{W} \subseteq \operatorname{Im}(\mathcal{P} p)$.

Theorem 5.5. If $p: Y \rightarrow X$ is a semicovering map, then $\pi^{\tau} p: \pi^{\tau} Y \rightarrow \pi^{\tau} X$ is an open covering morphism.

Proof. It has already been observed that $\pi^{\tau} p$ is both a covering morphism and a Topfunctor so it suffices to show $\pi^{\tau} p$ is open. Note that each function $\pi p: \pi Y\left(y_{1}, y_{2}\right) \rightarrow$ $\pi X\left(p\left(y_{1}\right), p\left(y_{2}\right)\right)$ is injective since $\pi p$ is a covering morphism of groupoids. This injectivity is independent of topologies on the hom-sets. We use the inductive approach to $\pi^{\tau}$ discussed in 4.7. For simplicity of notation, let $\mathcal{H}_{0}=\pi^{q t o p} Y$ and $\mathcal{G}_{0}=\pi^{q t o p} X$ and inductively take $\mathcal{H}_{\zeta}$ and $\mathcal{G}_{\zeta}$ to be the approximating qTop-groupoids of $\tau\left(\mathcal{H}_{0}\right)=\pi^{\tau} Y$ and $\tau\left(\mathcal{G}_{0}\right)=\pi^{\tau} X$ respectively. For the first inductive step, we show that whenever $p\left(y_{i}\right)=x_{i}, i=1,2$, the map $\pi p: \mathcal{H}_{0}\left(y_{1}, y_{2}\right) \rightarrow \mathcal{G}_{0}\left(x_{1}, x_{2}\right),[\alpha] \mapsto[p \circ \alpha]$ is open. 
Let $U$ be an open neighborhood in $\mathcal{H}_{0}\left(y_{1}, y_{2}\right)$. The diagram

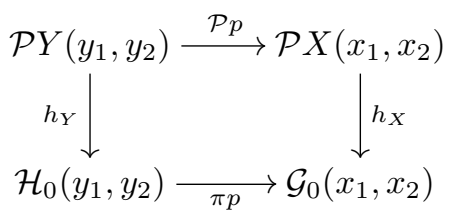

commutes when $h_{Y}$ and $h_{X}$ are the canonical quotient maps. Since $h_{X}$ is quotient and the top map is open by Lemma 5.4, it suffices to show $h_{X}^{-1}(\pi p(U))=\mathcal{P} p\left(h_{Y}^{-1}(U)\right)$. If $\alpha \in h_{X}^{-1}(\pi p(U))$, then $[\alpha] \in \pi p(U)$ and the lift $\tilde{\alpha}_{y_{1}}$ ends at $y_{2}$ by 3. of Lemma 2.4. Now that $\pi p\left(\left[\tilde{\alpha}_{y_{1}}\right]\right)=[\alpha] \in \pi p(U)$, the injectivity of $\pi p$ gives $\left[\tilde{\alpha}_{y_{1}}\right] \in U$. Therefore $\alpha=p \circ \tilde{\alpha}_{y_{1}}=\mathcal{P} p\left(\tilde{\alpha}_{y_{1}}\right)$ for $\tilde{\alpha}_{y_{1}} \in h_{Y}^{-1}(U)$. For the other inclusion, if $\alpha=p \circ \tilde{\alpha}_{y_{1}}$ such that $\left[\tilde{\alpha}_{y_{1}}\right] \in U$, then $[\alpha]=\pi p\left(\left[\tilde{\alpha}_{y_{1}}\right]\right) \in \pi p(U)$ and therefore $\alpha \in h_{X}^{-1}(\pi p(U))$.

Suppose $\zeta$ is an ordinal and that for each $\eta<\zeta, \pi p: \mathcal{H}_{\eta}\left(y_{1}, y_{2}\right) \rightarrow \mathcal{G}_{\eta}\left(p\left(y_{1}\right), p\left(y_{2}\right)\right)$ is open for each $y_{1}, y_{2} \in Y$. Fix $y_{1}, y_{2} \in Y$ and let $p\left(y_{i}\right)=x_{i}$. Clearly, if $\zeta$ is a limit ordinal, then $\pi p: \mathcal{H}_{\zeta}\left(y_{1}, y_{2}\right) \rightarrow \mathcal{G}_{\zeta}\left(x_{1}, x_{2}\right)$ is an open embedding. If $\zeta$ is a successor ordinal, consider the following diagram.

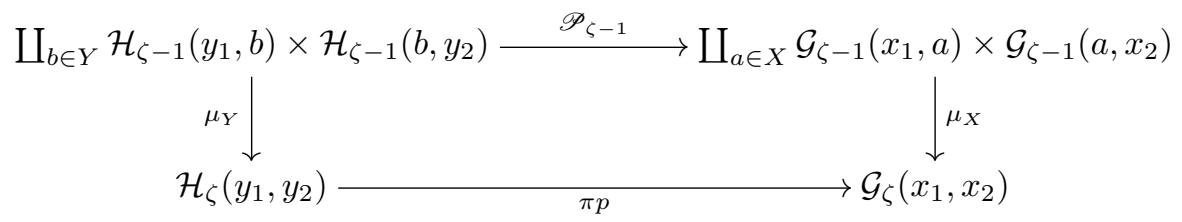

The vertical multiplication maps are quotient by definition. The top map $\mathscr{P}_{\zeta-1}$ is, on each summand, the product of open embeddings (by induction hypothesis):

$$
\pi p \times \pi p: \mathcal{H}_{\zeta-1}\left(y_{1}, b\right) \times \mathcal{H}_{\zeta-1}\left(b, y_{2}\right) \rightarrow \mathcal{G}_{\zeta-1}\left(x_{1}, p(b)\right) \times \mathcal{G}_{\zeta-1}\left(p(b), x_{2}\right),
$$

where $([\alpha],[\beta]) \mapsto([p \circ \alpha],[p \circ \beta])$. Therefore $\mathscr{P}_{\zeta-1}$ is continuous and open. Now suppose $U$ is open in $\mathcal{H}_{\zeta}\left(y_{1}, y_{2}\right)$. It suffices to show $\mu_{X}^{-1}(\pi p(U))$ is open. If $([\delta],[\epsilon]) \in$ $\mu_{X}^{-1}(\pi p(U))$, then $[\delta * \epsilon] \in \pi p(U)$. Let $a_{0}=\delta(1)$. Consequently, the lift of $\delta * \epsilon$ starting at $y_{1}$ ends at $y_{2}$. This lift is $\tilde{\delta}_{y_{1}} * \tilde{\epsilon}_{b_{0}}$, where $b_{0}=\tilde{\delta}_{y_{1}}(1) \in p^{-1}\left(a_{0}\right)$. Since

$$
\pi p\left(\left[\tilde{\delta}_{y_{1}} * \tilde{\epsilon}_{b_{0}}\right]\right)=\left[p \circ\left(\tilde{\delta}_{y_{1}} * \tilde{\epsilon}_{b_{0}}\right)\right]=\left[\left(p \circ \tilde{\delta}_{y_{1}}\right) *\left(p \circ \tilde{\epsilon}_{b_{0}}\right)\right]=[\delta * \epsilon] \in \pi p(U)
$$

and $\pi p$ is injective, $\left[\tilde{\delta}_{y_{1}} * \tilde{\epsilon}_{b_{0}}\right] \in U$. Therefore $\mu_{Y}^{-1}(U)$ is an open neighborhood of $\left(\left[\tilde{\delta}_{y_{1}}\right],\left[\tilde{\epsilon}_{b_{0}}\right]\right)$ and $\mathscr{P}_{\zeta-1}\left(\mu_{Y}^{-1}(U)\right)$ is an open neighborhood of $([\delta],[\epsilon])$ in

$$
\coprod_{a \in X} \mathcal{G}_{\zeta-1}\left(x_{1}, a\right) \times \mathcal{G}_{\zeta-1}\left(a, x_{2}\right)
$$

It then suffices to check the inclusion $\mathscr{P}_{\zeta-1}\left(\mu_{Y}^{-1}(U)\right) \subseteq \mu_{X}^{-1}(\pi p(U))$. This follows easily from noticing that if $([\alpha],[\beta]) \in \mu_{Y}^{-1}(U)$, then $[\alpha * \beta] \in U$ and

$$
\mu_{X}\left(\mathscr{P}_{\zeta-1}([\alpha],[\beta])\right)=[p \circ \alpha][p \circ \beta]=[p \circ(\alpha * \beta)]=\pi p([\alpha * \beta]) \in \pi p(U) .
$$

Corollary 5.6. If $p: Y \rightarrow X$ is a semicovering such that $p\left(y_{0}\right)=x_{0}$, the induced homomorphism $p_{*}: \pi_{1}^{\tau}\left(Y, y_{0}\right) \rightarrow \pi_{1}^{\tau}\left(X, x_{0}\right)$ is an open embedding of topological groups. 
We now see that for any space $X$, the Top-fundamental groupoid functor provides a functor $\pi^{\tau}: \operatorname{SCov}(X) \rightarrow \operatorname{OCovMor}\left(\pi^{\tau} X\right)$. Our main result is that, for all $X$ in the class of (locally wep-connected) spaces defined in the next section, $\pi^{\tau}$ is an equivalence of categories. The motivation for extending beyond locally path connected spaces to this class lies in potential applications to the theory of topological groups and Topgroupoids.

\section{Local properties of endpoints of paths}

It is unrealistic to expect that semicoverings of arbitrary spaces are classified by their induced covering morphisms; recall Zeeman's example mentioned in the introduction. Here, we specify exactly which spaces are to be included in our theory. The notions of wep- and local wep-connectedness defined below generalize local path connectedness. Definition 6.1 first appeared in [4] in the context of a van Kampen theorem for $\pi_{1}^{\tau}$.

Definition 6.1. Let $X$ be a space.

1. A path $\alpha: I \rightarrow X$ is well-ended if for every open neighborhood $\mathcal{U}$ of $\alpha$ in $\mathcal{P} X$ there are open neighborhoods $V_{0}, V_{1}$ of $\alpha(0), \alpha(1)$ in $X$ respectively such that for every $a \in V_{0}, b \in V_{1}$ there is a path $\beta \in \mathcal{U}$ with $\beta(0)=a$ and $\beta(1)=b$.

2. A path $\alpha: I \rightarrow X$ is well-targeted if for every open neighborhood $\mathcal{U}$ of $\alpha$ in $(\mathcal{P} X)_{\alpha(0)}$ there is an open neighborhood $V_{1}$ of $\alpha(1)$ such that for each $b \in V_{1}$, there is a path $\beta \in \mathcal{U}$ with $\beta(1)=y$.

A space $X$ is wep-connected if each pair of points can be connected by a well-ended path.

Some intuition for well-ended and well-targeted paths is given in [4].

Proposition 6.2. If $X$ is wep-connected and $x_{0} \in X$, then the evaluation map $e v_{1}:(\mathcal{P} X)_{x_{0}} \rightarrow X, \alpha \mapsto \alpha(1)$ is quotient.

Proof. Suppose $x \in U \subseteq X$ such that $e v_{1}^{-1}(U)$ is open in $(\mathcal{P} X)_{x_{0}}$. Since $X$ is wepconnected, there is a well-targeted path $\gamma \in(\mathcal{P} X)_{x_{0}}$ ending at $x$. Since $e v_{1}^{-1}(U)$ is an open neighborhood of $\gamma$, there is an open neighborhood $V$ of $x$ in $X$ such that for each $v \in V$ there is a path $\alpha \in e v_{1}^{-1}(U)$ from $x_{0}$ to $v$. Thus $V \subseteq U$.

Proposition 6.3. If the path components of $X$ are wep-connected, then $\pi_{0}^{q t o p} X$ is discrete, i.e., the path components of $X$ are open.

Proof. Let $x \in X$ and $\alpha$ be any well-ended path such that $\alpha(0)=x$. Since $\mathcal{P} X$ is an open neighborhood of $\alpha$, there are open neighborhoods $V_{0}, V_{1}$ of $x, \alpha(1)$ respectively such that for each $a \in V_{0}, b \in V_{1}$ there is a path $\gamma \in \mathcal{P} X$ from $a$ to $b$. Since for any $a \in V_{0}$, there is a path $\gamma$ from $a$ to $\alpha(1)$, it is clear that $V_{0}$ is contained in the path component of $x$.

The previous two propositions indicate that spaces with wep-connected path components are suitable for application of Lemma 2.5. Unfortunately, difficulties arise as one attempts to construct semicoverings of general wep-connected spaces. We are then motivated to slightly strengthen this notion. 
Definition 6.4. Let $X$ be a space.

1. A path $\alpha: I \rightarrow X$ is locally well-ended if for every open neighborhood $\mathcal{U}$ of $\alpha$ in $\mathcal{P} X$ there are open neighborhoods $V_{0}, V_{1}$ of $\alpha(0), \alpha(1)$ in $X$ respectively such that for every $a \in V_{0}, b \in V_{1}$ there is a well-ended path $\beta \in \mathcal{U}$ with $\beta(0)=a$ and $\beta(1)=b$.

2. A path $\alpha: I \rightarrow X$ is locally well-targeted if for every open neighborhood $\mathcal{U}$ of $\alpha$ in $(\mathcal{P} X)_{\alpha(0)}$ there is an open neighborhood $V_{1}$ of $\alpha(1)$ such that for each $b \in V_{1}$, there is a well-targeted path $\beta \in \mathcal{U}$ with $\beta(1)=y$.

A space $X$ is locally wep-connected if every pair of points in $X$ can be connected by a locally well-ended path.

Remark 6.5. The definitions of well-ended and well-targeted paths address the same property of the underlying space. It is shown in [4] that for fixed $x \in X, X$ is wepconnected if and only if for each $x^{\prime} \in X$, there is a well-targeted path from $x$ to $x^{\prime}$. The analogous statement holds for locally wep-connected spaces and locally well-targeted paths. We repeatedly call upon this fact without reference. In situations where it is necessary to use a basepoint, it is more convenient to work with well-targeted and locally well-targeted paths.

Clearly every locally wep-connected space is wep-connected. Examples of spaces which are wep-connected but not locally wep-connected exist but are complicated and would distract from our purposes. Additionally, if $\alpha: I \rightarrow X$ is a path and $X$ is locally path connected at $\alpha(0)$ and $\alpha(1)$, then $\alpha$ is well-ended. It follows immediately that any path connected, locally path connected space is locally wep-connected. There are many non-locally path connected spaces which are locally wep-connected (for instance, see Prop. 6.7 below).

Working with (locally) well-ended paths requires working extensively with the compact-open topology. We therefore make heavy use of the following machinery (as is used in $[3,4]$ ) for dealing with operations on neighborhoods of paths.

Recall the notation for paths introduced at the beginning of Section 2. Let $\mathcal{U}=\bigcap_{j=1}^{n}\left\langle C_{j}, U_{j}\right\rangle$ be an open neighborhood of a path $p \in \mathcal{P} X$ and $A \subseteq I$ be a closed subinterval. Then

$$
\mathcal{U}_{A}=\bigcap_{A \cap C_{j} \neq \emptyset}\left\langle T_{A}^{-1}\left(A \cap C_{j}\right), U_{j}\right\rangle
$$

is an open neighborhood of $p_{A}$. If $A=\{t\}$ is a singleton, let $\mathcal{U}_{A}=\left\langle I, \bigcap_{t \in C_{j}} U_{j}\right\rangle$. On the other hand, if $p=q_{A}$ for some path $q \in \mathcal{P} X$, then $\mathcal{U}^{A}=\bigcap_{j=1}^{n}\left\langle T_{A}\left(C_{j}\right), U_{j}\right\rangle$ is an open neighborhood of $q$. If $A=\{t\}$ so that $p_{A}=c_{p(t)}$, let $\mathcal{U}^{A}=\bigcap_{j=1}^{n}\left\langle\{t\}, U_{j}\right\rangle$. The following observation illustrates how one may "place one neighborhood after another" using this notation.

Lemma 6.6. Let $\mathcal{U}=\bigcap_{j=1}^{n}\left\langle C_{j}, U_{j}\right\rangle$ be an open neighborhood in $\mathcal{P} X$ such that $\bigcup_{j=1}^{n} C_{j}=I$. Then

1. For any closed interval $A \subseteq I,\left(\mathcal{U}^{A}\right)_{A}=\mathcal{U} \subseteq\left(\mathcal{U}_{A}\right)^{A}$.

2. If $0=t_{0} \leqslant t_{1} \leqslant t_{2} \leqslant \ldots \leqslant t_{n}=1$, then $\mathcal{U}=\bigcap_{i=1}^{n}\left(\mathcal{U}_{\left[t_{i-1}, t_{i}\right]}\right)^{\left[t_{i-1}, t_{i}\right]}$. 
For brevity, if $\mathcal{U}=\bigcap_{i=1}^{m}\left\langle C_{i}, U_{i}\right\rangle$ and $\mathcal{V}=\bigcap_{j=1}^{n}\left\langle D_{j}, V_{j}\right\rangle$ are neighborhoods of $\alpha$ and $\beta$ respectively where $\alpha(1)=\beta(0)$, we write $\mathcal{U} \mathcal{V}$ for the neighborhood $\mathcal{U}^{\left[0, \frac{1}{2}\right]} \cap \mathcal{V}^{\left[\frac{1}{2}, 1\right]}$ of $\alpha * \beta$.

The generalized wedge of circles on the topological space $X$ is the quotient

$$
\Sigma\left(X_{+}\right)=\frac{X \times I}{X \times\{0,1\}}
$$

which can be thought of as a wedge of circles parameterized by $X$. These spaces, studied in detail in [3], are typically not locally path connected, however, the topological fundamental group $\pi_{1}^{\tau}\left(\Sigma\left(X_{+}\right), x_{0}\right)$ is naturally isomorphic to the free topological group $F_{M}\left(\pi_{0}^{q t o p}(X)\right)$ : see [4]. It is therefore desirable that semicovering theory applies to such spaces.

Proposition 6.7. For every space $X$, the generalized wedge of circles $\Sigma\left(X_{+}\right)$is locally wep-connected. Moreover, a space obtained by attaching $n$-cells, $n \geqslant 2$ to $\Sigma\left(X_{+}\right)$is locally wep-connected.

Proof. Since $\Sigma\left(X_{+}\right)$is locally path connected at its basepoint $x_{0}$, pick $x \wedge t \in X \wedge$ $(0,1)$. Let $\alpha$ be any path from $x_{0}$ to $x \wedge t$ satisfying $\alpha(s)=x \wedge r(s)$, and $\alpha^{-1}\left(x_{0}\right)=$ $\{0\}$. A basic open neighborhood of $\alpha$ may be taken to be of the form

$$
\mathcal{U}=\left\langle K_{n}^{1}, V\right\rangle \cap \bigcap_{j=2}^{n}\left\langle K_{n}^{j}, U \wedge\left(a_{j}, b_{j}\right)\right\rangle,
$$

where $V$ is a basic neighborhood of $x_{0}$ and $U$ is an open neighborhood of $x$ in $X$. If $u \wedge v \in U \wedge\left(a_{n}, b_{n}\right)$, then the path $\beta(s)=u \wedge r(s)$ from $x_{0}$ to $u \wedge t$ lies in $\mathcal{U}$ and also satisfies $\beta^{-1}\left(x_{0}\right)=\{0\}$. Let $\gamma$ be the arc $\gamma(s)=u \wedge(t+s(v-t))$ in $\{u\} \wedge\left(a_{n}, b_{n}\right)$ from $u \wedge t$ to $u \wedge v$ and $\delta$ be the path satisfying $\delta_{\left[0, \frac{2 n-1}{2 n}\right]}=\beta$ and $\delta_{K_{2 n}^{2 n}}=\gamma$. Clearly $\delta \in \mathcal{U}$ is a path from $x_{0}$ to $u \wedge v$ and therefore $\alpha$ is well-targeted. Since $\delta$ is also of the form $\delta(s)=y \wedge r^{\prime}(s)$ where $\delta^{-1}\left(x_{0}\right)=\{0\}$, it follows that $\delta$ is well-targeted. Thus $\alpha$ is locally well-targeted. Since n-cells are locally path connected, a straightforward extension of this argument applies to spaces obtained by attaching cells to $\Sigma\left(X_{+}\right)$.

Corollary 6.8. For any space $\left(Y, y_{0}\right)$, there is a locally wep-connected space $X$ and a map $X \rightarrow Y$ which induces an isomorphism $\pi_{1}^{\tau}\left(X, x_{0}\right) \rightarrow \pi_{1}^{\tau}\left(Y, y_{0}\right)$ of topological groups.

Proof. The based counit $c u: \Sigma\left(\Omega\left(Y, y_{0}\right)_{+}\right) \rightarrow Y$ of the loop-suspension adjunction induces a quotient map $c u_{*}: \pi_{1}^{\tau}\left(\Sigma\left(\Omega\left(Y, y_{0}\right)_{+}\right), x_{0}\right) \rightarrow \pi_{1}^{\tau}\left(Y, y_{0}\right)$ of topological groups. For each $[\beta] \in \operatorname{ker}\left(c u_{*}\right)$, attach a 2-cell using a representative loop

$$
\beta: S^{1} \rightarrow \Sigma\left(\Omega\left(Y, y_{0}\right)_{+}\right) .
$$

Let $X$ be the resulting space, which is locally wep-connected by the previous proposition. The inclusion $j: \Sigma\left(\Omega\left(Y, y_{0}\right)_{+}\right) \rightarrow X$ induces a quotient map

$$
j_{*}: \pi_{1}^{\tau}\left(\Sigma\left(\Omega\left(Y, y_{0}\right)_{+}\right), x_{0}\right) \rightarrow \pi_{1}^{\tau}\left(X, x_{0}\right)
$$

of topological groups [4]. Since $\operatorname{ker}\left(j_{*}\right)=\operatorname{ker}\left(c u_{*}\right)$, there is a based map $X \rightarrow Y$ which induces the desired isomorphism. 
The following lemma is straightforward when the above machinery for the compactopen topology is used.

Lemma 6.9. Let $X$ be a space and $\alpha: I \rightarrow X$ be a path.

1. If there is $a 0 \leqslant t \leqslant 1$ such that $\alpha_{[t, 1]}$ is well-targeted (resp. locally well-targeted), then $\alpha$ is well-targeted (resp. locally well-targeted).

2. If there are $0 \leqslant s \leqslant t \leqslant 1$ such that $\alpha_{[t, 1]}$ and $\left(\alpha_{[0, s]}\right)^{-1}$ are well-targeted (resp. locally well-targeted), then $\alpha$ is well-ended (resp. locally well-ended).

3. The reverse of a well-ended (resp. locally well-ended) path is well-ended (resp. locally well-ended).

4. The concatenation of well-ended (resp. locally well-ended) paths is well-ended (resp. locally well-ended).

Proof. 1. If $\alpha_{[t, 1]}$ is well-targeted and $\mathcal{U}$ is an open neighborhood of $\alpha$, we find a neighborhood $\mathcal{V}$ of $\alpha$ of the form $\mathcal{V}=\bigcap_{j=1}^{n}\left\langle K_{n}^{j}, U_{j}\right\rangle$ contained in $\mathcal{U}$. Now $\mathcal{V}_{[t, 1]}$ is an open neighborhood of $\alpha_{[t, 1]}$. Note that if $t=1, \alpha_{[t, 1]}$ is constant at $\alpha(1)$, we may take $\mathcal{V}_{[t, 1]}=\left\langle I, U_{n}\right\rangle$. By assumption, there is an open neighborhood $V$ of $\alpha(1)$ contained in $U_{n}$ such that for each $v \in V$ there is a path $\gamma \in \mathcal{V}_{[t, 1]}$ from $\alpha(t)$ to $v$. When $t \neq 1$, the path $\beta$ satisfying $\beta_{[0, t]}=\alpha_{[0, t]}$ and $\beta_{[t, 1]}=\gamma$ is the desired path in $\mathcal{V}$ (and thus in $\mathcal{U}$ ) from $\alpha(0)$ to $v$. When $t=1$, let $\beta$ be the path satisfying $\beta_{\left[0, \frac{n-1}{n}\right]}=\alpha_{\left[0, \frac{n-1}{n}\right]}$, $\beta_{K_{2 n}^{2 n-1}}=\alpha_{K_{n}^{n}}$, and $\beta_{K_{2 n}^{2 n}}=\gamma$. Now $\beta$ is a path in $\mathcal{V}$ from $\alpha(0)$ to $v$. In the case that $\alpha_{[t, 1]}$ is locally well-targeted and $t \neq 1$, we take $\gamma$ in the previous argument to be welltargeted. Since $\beta \in \mathcal{U}$ is such that $\beta_{[t, 1]}=\gamma$ is well-targeted, $\beta$ itself is well-targeted and $\alpha$ is locally well-targeted. Similarly, when $t \neq 1$, we have $s=\frac{2 n-1}{2 n}<1$ such that $\beta_{[0, s]}=\gamma$ is well-targeted. Thus $\beta$ is well-targeted and $\alpha$ is locally well-targeted.

2 . follows from the same type of argument used in 1 .

3. follows from the fact that $\delta^{-1} \in \mathcal{V}$ if and only if $\delta \in \mathcal{V}^{-1}$.

4. follows directly from 2 .

The following Corollary allows us to replace any path in a locally wep-connected space by a homotopic (rel. endpoints) locally well-targeted path.

Corollary 6.10. Let $X$ be wep-connected (resp. locally wep-connected) and $x_{1}, x_{2} \in$ $X$. For each class $[\alpha] \in \pi X\left(x_{1}, x_{2}\right)$, there is a well-targeted (resp. locally well-targeted) path $\beta \in[\alpha]$.

Proof. If $X$ is wep-connected (resp. locally wep-connected), there is a well-targeted (resp. locally well-targeted) path $\gamma$ from $x_{1}$ to $x_{2}$. Let $\beta=\alpha * \gamma^{-1} * \gamma$. Clearly $\alpha \simeq \beta$ and 1. of Lemma 6.9 implies that $\beta$ is well-targeted (resp. locally well-targeted).

The next two statements are motivated by the desire to lift properties of a space to its semicoverings.

Proposition 6.11. Let $p: Y \rightarrow X$ be a semicovering map such that $p\left(y_{0}\right)=x_{0}$. If $\alpha \in(\mathcal{P} X)_{x_{0}}$ is (locally) well-targeted, then so is the lift $\tilde{\alpha}_{y_{0}}$.

Proof. Suppose $\alpha$ is well-targeted and let $\mathcal{W}$ be an open neighborhood of $\tilde{\alpha}_{y_{0}}$ in $(\mathcal{P} Y)_{y_{0}}$. Since $p$ is a local homeomorphism, there is an open neighborhood $U$ of 
$\tilde{\alpha}_{y_{0}}(1)$ mapped homeomorphically by $p$ onto an open subset of $X$. Let $\mathcal{U}=\mathcal{W} \cap$ $\langle\{1\}, U\rangle$. Since $\mathcal{P} p:(\mathcal{P} Y)_{y_{0}} \rightarrow(\mathcal{P} X)_{x_{0}}$ is a homeomorphism, $\mathcal{V}=\mathcal{P} p(\mathcal{U})$ is an open neighborhood of $\alpha$. By assumption, there is an open neighborhood $V$ of $\alpha(1)$ (which we may take to be contained in $p(U)$ ) such that for each $v \in V$ there is a path $\gamma \in \mathcal{V}$ from $x_{0}$ to $v$. Now $W=p^{-1}(V) \cap U$ is a homeomorphic copy of $V$ in $U$. If $w \in W$, then $p(w) \in V$ and there is a path $\gamma \in \mathcal{V}$ from $x_{0}$ to $p(w)$. Since $L_{p}: \mathcal{V} \cong \mathcal{U}$, the lift $\tilde{\gamma}_{y_{0}}$ of $\gamma$ lies in $\mathcal{U}$. Since $p \circ \tilde{\gamma}_{y_{0}}(1)=p(w)$ and $\tilde{\gamma}_{y_{0}}(1) \in p^{-1}(p(w)) \cap U=\{w\}$, we have $\tilde{\gamma}_{y_{0}}(1)=w$. Since we have already shown that lifts of well-targeted paths are welltargeted, the locally well-targeted case follows from the same argument and taking $\gamma$ to be well-targeted.

Corollary 6.12. Let $p: Y \rightarrow X$ be a semicovering map. If $X$ is locally path connected, then so is $Y$. If $X$ is wep-connected (resp. locally wep-connected), then so is every path component of $Y$.

Proof. The locally path connected case is clear since $p$ is a local homeomorphism. For the other two cases we let $p\left(y_{0}\right)=x_{0}$ and show the path component of $y_{0}$ in $Y$ is wep-connected (resp. locally wep-connected). Suppose $y \in Y, p(y)=x$, and $\tilde{\gamma}_{y_{0}}$ is a path from $y_{0}$ to $y$ so that $\gamma=p \circ \tilde{\gamma}_{y_{0}}$ is a path from $x_{0}$ to $x$. By Corollary 6.10 , there is a well-targeted (resp. locally well-targeted) path $\alpha$ from $x_{0}$ to $x$ homotopic to $\gamma$. This homotopy lifts to a homotopy of paths $\tilde{\gamma}_{y_{0}} \simeq \tilde{\alpha}_{y_{0}}$. In particular, $\tilde{\alpha}_{y_{0}}(1)=\tilde{\gamma}_{y_{0}}(1)=y$ and $\tilde{\alpha}_{y_{0}}$ is well-targeted (resp. locally well-targeted) by Proposition 6.11.

\section{Classification Theorems}

The classification of semicoverings is stated conveniently in the following theorem.

Theorem 7.1. For a locally wep-connected space $X$, the functor

$$
\pi^{\tau}: \operatorname{SCov}(X) \rightarrow \operatorname{OCovMor}\left(\pi^{\tau} X\right), p \mapsto \pi^{\tau} p
$$

is an equivalence of categories.

The main difficulty in the proof of this theorem is the existence of a semicovering $p$ whose induced covering morphism $\pi^{\tau} p$ is equivalent to a given open covering morphism $\mathcal{H} \rightarrow \pi^{\tau} X$. Existence is dealt with in Section 7.1 and the proof is completed in 7.2. Theorem 7.1 is extended easily to include topological sums of locally wep-connected spaces, i.e., spaces whose path components are locally wep-connected. Under the usual conditions of covering space theory, this classification reduces to the classification of coverings.

Corollary 7.2. If $X$ is locally path connected and semilocally 1-connected, then $\operatorname{Cov}(X) \simeq \operatorname{SCov}(X)$ and $\operatorname{Cov}_{\mathbf{0}}(X) \simeq \operatorname{SCov}_{\mathbf{0}}(X)$.

Proof. For any such $X$ and $x_{0} \in X, \pi_{1}^{\tau}\left(X, x_{0}\right)$ is a discrete group [4]. Thus $\pi^{\tau} X$ is a discrete Top-groupoid. This gives the middle equivalence in the following factorization of the inclusion.

$$
\operatorname{SCov}(X) \simeq \operatorname{OCovMor}\left(\pi^{\tau} X\right) \simeq \operatorname{CovMor}(\pi X) \simeq \operatorname{Cov}(X)
$$

It remains to be seen if there are more general conditions guaranteeing $\mathbf{S C o v}(X) \simeq$ $\operatorname{Cov}(X)$. 


\subsection{Existence of semicoverings}

Let $X$ be a path connected space and $F: \mathcal{H} \rightarrow \pi X$ be a covering morphism of groupoids such that $O b(\mathcal{H}) \neq \emptyset$. The two conditions here imply that $O b(F)$ is surjective. For each $y \in O b(F)^{-1}(x)$ and $\alpha \in(\mathcal{P} X)_{x}$, let $y \cdot[\alpha]$ denote the target of the unique morphism $\widetilde{[\alpha]_{y}} \in \mathcal{H}_{y}$ such that $F\left(\widetilde{[\alpha]_{y}}\right)=[\alpha] \in(\pi X)_{x}$. Let $\tilde{X}_{F}$ be the space obtained by giving the set $O b(\mathcal{H})$ the quotient topology with respect to the map

$$
\Theta_{F}: \coprod_{x \in X} O b(F)^{-1}(x) \times(\mathcal{P} X)_{x} \rightarrow O b(\mathcal{H}) \text { where } \Theta_{F}(y, \alpha)=y \cdot[\alpha] .
$$

Here each fiber $O b(F)^{-1}(x)$ in the topological sum has the discrete topology. Since the quotient topology on $\tilde{X}_{F}$ is characterized by the quotient map $\Theta_{F}$, we sometimes write a generic element of $\tilde{X}_{F}$ as $y \cdot[\alpha]$. Let

$$
p_{F}=O b(F): \tilde{X}_{F} \rightarrow X .
$$

Since $p_{F}(y \cdot[\alpha])=\alpha(1)$, the diagram

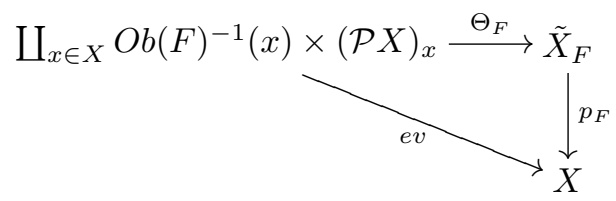

commutes when $e v$ is evaluation $e v(y, \alpha)=\alpha(1)$. Thus $p_{F}$ is continuous by the universal property of quotient spaces.

Proposition 7.3. If $F^{\prime}: \mathcal{H}^{\prime} \rightarrow \pi X$ is another covering morphism and $K: \mathcal{H} \rightarrow \mathcal{H}^{\prime}$ is a functor such that $F^{\prime} \circ K=F$, then $\mathrm{Ob}(K): \tilde{X}_{F} \rightarrow \tilde{X}_{F^{\prime}}$ is continuous.

Proof. For each $x \in X$, let $K_{x}: O b(F)^{-1}(x) \rightarrow O b\left(F^{\prime}\right)^{-1}(x)$ be the restriction of $O b(K)$ to $O b(F)^{-1}(x)$. Since $F^{\prime} \circ K=F$ where $F, F^{\prime}$ are covering morphisms, we have $K(y \cdot[\alpha])=K_{x}(y) \cdot[\alpha]$ whenever $F(y)=x$ and $\alpha \in(\mathcal{P} X)_{x}$. Thus the following diagram commutes:

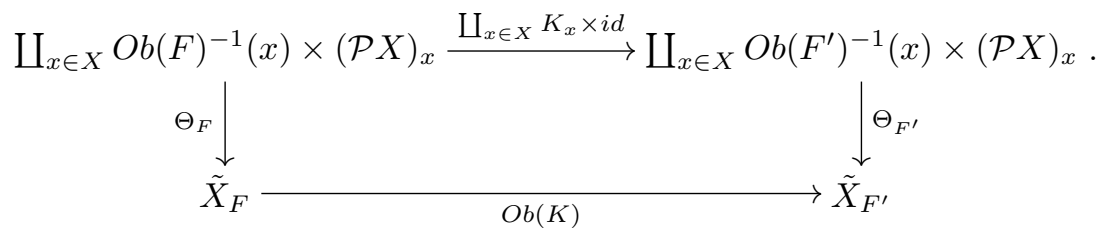

The top horizontal map is continuous since each fiber $O b(F)^{-1}(x)$ is discrete. The continuity of the bottom map then follows from the universal property of quotient spaces.

Proposition 7.4. If $X$ is wep-connected, then $p_{F}: \tilde{X}_{F} \rightarrow X$ is open.

Proof. Suppose $W$ is open in $\tilde{X}_{F}$ and $x=p_{F}(y) \in p_{F}(W)$ for $y \in W$. Note that $y \cdot\left[c_{x}\right]=y$. By Corollary 6.10 , there is a well-targeted, null-homotopic loop $\beta \in$ $\Omega(X, x)$. Thus $y \cdot[\beta]=y \cdot\left[c_{x}\right]=y \in W$. Now $\{y\} \times \mathcal{U}=\Theta_{F}^{-1}(W) \cap\left(\{y\} \times(\mathcal{P} X)_{x}\right)$ for some open neighborhood $\mathcal{U}$ of $\beta$ in $(\mathcal{P} X)_{x}$. Since $\beta$ is well-targeted, there is an open neighborhood $V$ of $x=\beta(1)$ in $X$ such that for every $v \in V$, there is a path 
$\delta \in \mathcal{U}$ such that $\delta(1)=v$. Thus $y \cdot[\delta] \in W$ and $p_{F}(y \cdot[\delta])=\delta(1)=v$. This gives the inclusion $V \subseteq p_{F}(W)$.

Canonical lifts of paths 7.5. Without any other assumptions on $X$ or $F$, we find canonical lifts of paths with respect to $p_{F}$. For $y \in p_{F}^{-1}(x)$, we construct a canonical section to

$$
\mathcal{P} p_{F}:\left(\mathcal{P} \tilde{X}_{F}\right)_{y} \rightarrow(\mathcal{P} X)_{x}
$$

as the composition of continuous functions. Since multiplication $\mu: I \times I \rightarrow I$ of real numbers is continuous,

$$
\mu^{\#}:(\mathcal{P} X)_{x} \rightarrow \operatorname{Top}((I \times I,\{0\} \times I \cup I \times\{0\}),(X,\{x\})), \beta \mapsto \beta \circ \mu
$$

to the relative mapping space is continuous. Additionally,

$$
r: \operatorname{Top}((I \times I,\{0\} \times I \cup I \times\{0\}),(X,\{x\})) \rightarrow\left(\mathcal{P}(\mathcal{P} X)_{x}\right)_{c_{x}},
$$

where $r(\phi)(s)(t)=\phi(s, t)$ is a homeomorphism. Note that $r(\beta \circ \mu)(s)(t)=\beta(s t)$ and therefore $r(\beta \circ \mu)(s)=\beta_{[0, s]}$. Lastly, the map $\mathcal{P} \Theta_{F}:\left(\mathcal{P}(\mathcal{P} X)_{x}\right)_{c_{x}} \rightarrow\left(\mathcal{P} \tilde{X}_{F}\right)_{y}$ is obtained by applying $\mathcal{P}$ to the restriction of $\Theta_{F}$ to $\{y\} \times(\mathcal{P} X)_{x}$. Now let $L_{F}:(\mathcal{P} X)_{x} \rightarrow$ $\left(\mathcal{P} \tilde{X}_{F}\right)_{y}$ be the composition $\mathcal{P} \Theta_{F} \circ r \circ \mu^{\#}$ which takes $\beta$ to the path $\tilde{\beta}_{y}(s)=y$. $\left[\beta_{[0, s]}\right]$. Since $p_{F}\left(y \cdot\left[\beta_{[0, s]}\right]\right)=\beta(s), \tilde{\beta}_{y}$ is a lift of $\beta$ starting at $y$. Therefore, $L_{F}$ is the desired section.

Canonical lifts of homotopies 7.6. We take a similar approach to find canonical lifts of homotopies of paths by constructing a section of

$$
\Phi p_{F}:\left(\Phi \tilde{X}_{F}\right)_{y} \rightarrow(\Phi X)_{x}
$$

for $y \in p_{F}^{-1}(x)$. Since multiplication $m: I \times \Delta_{2} \rightarrow \Delta_{2}, m(s, t, u)=(s t, u)$ is continuous,

$$
m^{\#}:(\Phi X)_{x} \rightarrow \operatorname{Top}\left(\left(I \times \Delta_{2}, I \times e_{1} \cup \Delta_{2} \times\{0\}\right),(X, x)\right)
$$

given by $m^{\#}(\phi)(s, t, u)=\phi(s t, u)$ is continuous. Additionally, the map

$$
r: \operatorname{Top}\left(\left(I \times \Delta_{2}, I \times e_{1} \cup \Delta_{2} \times\{0\}\right),(X, x)\right) \rightarrow\left(\Phi(\mathcal{P} X)_{x}\right)_{c_{x}}
$$

given by $r(K)(t, u)(s)=K(s, t, u)$ is a homeomorphism. Note that $(r(\phi \circ m)(x, y))(s)$ $=\phi(s x, y)$. Additionally, $\Phi \Theta_{F}:\left(\Phi(\mathcal{P} X)_{x}\right)_{c_{x}} \rightarrow\left(\Phi \tilde{X}_{F}\right)_{y}$ is obtained by applying $\Phi$ to the restriction of $\Theta_{F}$ to $\{y\} \times(\mathcal{P} X)_{x}$ and $h L_{F}:(\Phi X)_{x} \rightarrow\left(\Phi \tilde{X}_{F}\right)_{y}$ is the composition $\Phi \Theta_{F} \circ r \circ m^{\#}$. To see that $h L_{F}$ is a section of $\Phi p_{F}$ we check that $p_{F}\left(h L_{F}(\phi)(t, u)\right)=$ $\phi(t, u)$ for $(t, u) \in \Delta_{2}$. This is straightforward from the equation

$$
p_{F}\left(\Theta_{F}(r(\phi \circ m)(t, u))\right)=(r(\phi \circ m)(t, u))(1)=\phi(1 t, u)=\phi(t, u) .
$$

Theorem 7.7. The map $p_{F}: \tilde{X}_{F} \rightarrow X$ has continuous lifting of paths and homotopies if and only if $p_{F}$ has unique path lifting. 
Proof. According to 7.5 and 7.6 , for any $y \in p_{F}^{-1}(x)$ both

$$
\mathcal{P} p_{F}:\left(\mathcal{P} \tilde{X}_{F}\right)_{y} \rightarrow(\mathcal{P} X)_{x} \text { and } \Phi p_{F}:\left(\Phi \tilde{X}_{F}\right)_{y} \rightarrow(\Phi X)_{x}
$$

are topological retractions. Unique path lifting implies that both of these maps are injective and therefore homeomorphisms.

Remark 7.8. For $y_{1}, y_{2} \in \tilde{X}_{F}$, let $F \mathcal{H}\left(y_{1}, y_{2}\right)$ be the image of the injection $F: \mathcal{H}\left(y_{1}, y_{2}\right) \rightarrow \pi X\left(x_{1}, x_{2}\right)$. Since $F$ is a covering morphism,

$$
F \mathcal{H}\left(y_{1}, y_{2}\right)=\left\{[\alpha] \in \pi X\left(x_{1}, x_{2}\right) \mid y_{1} \cdot[\alpha]=y_{2}\right\}
$$

whenever $p_{F}\left(y_{i}\right)=x_{i}$. The case $y_{1}=y=y_{2}$ gives that $y \cdot[\alpha]=y \cdot[\beta]$ if and only if $\left[\alpha * \beta^{-1}\right] \in F \mathcal{H}(y)$.

From now on, we suppose $X$ is locally wep-connected, $\mathcal{H}$ is a Top-groupoid, and the covering morphism $F: \mathcal{H} \rightarrow \pi^{\tau} X$ is an open Top-functor. We require these assumptions to obtain a simple basis for the topology of $\tilde{X}_{F}$. Since each map $F: \mathcal{H}\left(y_{1}, y_{2}\right) \rightarrow$ $\pi^{\tau} X\left(x_{1}, x_{2}\right)$ is an open embedding, $F \mathcal{H}\left(y_{1}, y_{2}\right)$ is open in $\pi^{\tau} X\left(x_{1}, x_{2}\right)$. Additionally, the continuity of the maps $h: \mathcal{P} X\left(x_{1}, x_{2}\right) \rightarrow \pi^{\tau} X\left(x_{1}, x_{2}\right)$ identifying homotopy classes gives that the pre-image $h^{-1}\left(F \mathcal{H}\left(y_{1}, y_{2}\right)\right)$ is open in $\mathcal{P} X\left(x_{1}, x_{2}\right)$.

$A$ basis for the topology of $\tilde{X}_{F}$ 7.9. Suppose $p_{F}\left(y_{0}\right)=x_{0}, \alpha \in(\mathcal{P} X)_{x_{0}}$, and $U$ is an open neighborhood of $y_{0} \cdot[\alpha]$ in $\tilde{X}_{F}$. By Corollary 6.10 , we may assume that $\alpha$ is locally well-targeted. Note that $h^{-1}\left(F \mathcal{H}\left(y_{0}\right)\right)$ is an open neighborhood of the nullhomotopic loop $\alpha * \alpha^{-1}$ in $\Omega\left(X, x_{0}\right)$. This means we can find a neighborhood $\mathcal{U}=$ $\bigcap_{i=1}^{m}\left\langle K_{m}^{i}, A_{i}\right\rangle$ of $\alpha$ in $(\mathcal{P} X)_{x_{0}}$ such that

1. $\left\{y_{0}\right\} \times \mathcal{U} \subseteq \Theta_{F}^{-1}(U)$

2. $\alpha * \alpha^{-1} \in \mathcal{U U}^{-1} \cap \Omega\left(X, x_{0}\right) \subseteq h^{-1}\left(F \mathcal{H}\left(y_{0}\right)\right)$.

Since $\alpha$ is locally well-targeted, there is an open neighborhood $V$ of $\alpha(1)$ contained in $A_{m}$ such that for each $v \in V$, there is a well-targeted path $\delta \in \mathcal{U}$ from $x$ to $v$. Let

$$
B\left(y_{0} \cdot[\alpha], \mathcal{U}, V\right)=\Theta_{F}\left(\left\{y_{0}\right\} \times(\mathcal{U} \cap\langle\{1\}, V\rangle)\right) .
$$

Lemma 7.10. Sets of the form $B\left(y_{0} \cdot[\alpha], \mathcal{U}, V\right)$ give a basis for the topology of $\tilde{X}_{F}$. Moreover, $B\left(y_{0} \cdot[\alpha], \mathcal{U}, V\right)$ is mapped homeomorphically onto $V$ by $p_{F}$.

Proof. Since $U$ is arbitrary and $B\left(y_{0} \cdot[\alpha], \mathcal{U}, V\right) \subseteq U$, it suffices to show that $B\left(y_{0}\right.$. $[\alpha], \mathcal{U}, V)$ is open in $\tilde{X}_{F}$, or equivalently that $\Theta_{F}^{-1}\left(B\left(y_{0} \cdot[\alpha], \mathcal{U}, V\right)\right)$ is open. If

$$
\left(y_{1}, \beta\right) \in \Theta_{F}^{-1}\left(B\left(y_{0} \cdot[\alpha], \mathcal{U}, V\right)\right) \cap\left(\left\{y_{1}\right\} \times(\mathcal{P} X)_{x_{1}}\right),
$$

then $y_{1} \cdot[\beta]=y_{0} \cdot[\epsilon]$ for $\epsilon \in \mathcal{U} \cap\langle\{1\}, V\rangle$. Recall that this implies $\beta(1)=\epsilon(1)$. By assumption, there is a well-targeted path $\delta \in \mathcal{U}$ such that $\delta(1)=\epsilon(1)$. Since $\delta * \epsilon^{-1} \in \mathcal{U U}^{-1} \cap \Omega\left(X, x_{0}\right) \subseteq h^{-1}\left(F \mathcal{H}\left(y_{0}\right)\right)$, we have $y_{0} \cdot[\delta]=y_{0} \cdot[\epsilon]=y_{1} \cdot[\beta]$. Since $\left[\delta * \beta^{-1}\right] \in F \mathcal{H}\left(y_{0}, y_{1}\right)$, the set $h^{-1}\left(F \mathcal{H}\left(y_{0}, y_{1}\right)\right)$ is an open neighborhood of $\delta * \beta^{-1}$ in $\mathcal{P} X\left(x_{0}, x_{1}\right)$. This observation guarantees that there are open neighborhoods $\mathcal{B}=\bigcap_{j=1}^{n}\left\langle K_{n}^{j}, B_{j}\right\rangle$ of $\beta$ in $(\mathcal{P} X)_{x_{1}}$ and $\mathcal{D}=\bigcap_{k=1}^{p}\left\langle K_{p}^{k}, D_{k}\right\rangle$ of $\delta$ in $(\mathcal{P} X)_{x_{0}}$ such that

1. $\mathcal{D} \subseteq \mathcal{U}$

2. $\delta * \beta^{-1} \in \mathcal{D B}^{-1} \cap \mathcal{P} X\left(x_{0}, x_{1}\right) \subseteq h^{-1}\left(\mathcal{H}\left(y_{0}, y_{1}\right)\right)$ 
3. $B_{n} \cup D_{p} \subseteq V$.

Since $\delta$ is well-targeted, there is an open neighborhood $W$ of $\delta(1)=\beta(1)$ in $B_{n} \cap D_{p}$ such that for each $w \in W$, there is a path $\zeta \in \mathcal{D}$ from $x_{0}$ to $w$. We claim the neighborhood $\left\{y_{1}\right\} \times(\mathcal{B} \cap\langle\{1\}, W\rangle)$ of $\left(y_{1}, \beta\right)$ is contained in $\Theta_{F}^{-1}\left(B\left(y_{0} \cdot[\alpha], \mathcal{U}, V\right)\right) \cap$ $\left(\left\{y_{1}\right\} \times(\mathcal{P} X)_{x_{1}}\right)$. If $\gamma \in \mathcal{B} \cap\langle\{1\}, W\rangle$, there is a path $\zeta \in \mathcal{D}$ from $x_{0}$ to $\gamma(1)$. This gives $\zeta * \gamma^{-1} \in \mathcal{D B}^{-1} \cap \mathcal{P} X\left(x_{0}, x_{1}\right) \subseteq h^{-1}\left(F \mathcal{H}\left(y_{0}, y_{1}\right)\right)$ and therefore $y_{0} \cdot[\zeta]=$ $y_{1} \cdot[\gamma]$. Since $y_{1} \cdot[\gamma]=y_{0} \cdot[\zeta]$ for

$$
\zeta \in \mathcal{D} \cap\langle\{1\}, W\rangle \subseteq \mathcal{U} \cap\langle\{1\}, V\rangle,
$$

we have $y_{1} \cdot[\gamma] \in B\left(y_{0} \cdot[\alpha], \mathcal{U}, V\right)$.

Since $p_{F}$ is open by 7.4 , the restriction $B\left(y_{0} \cdot[\alpha], \mathcal{U}, V\right) \rightarrow V$ of $p_{F}$ is a homeomorphism if it is bijective. If $v \in V$, there is a path $\delta \in \mathcal{U}$ such that $\delta(1)=v$ which gives $p_{F}\left(y_{0} \cdot[\delta]\right)=v$. Additionally, if $\delta, \epsilon \in \mathcal{U} \cap\langle\{1\}, V\rangle$ such that $p_{F}\left(y_{0} \cdot[\delta]\right)=$ $\delta(1)=\epsilon(1)=p_{F}\left(y_{0} \cdot[\epsilon]\right)$, then $\delta * \epsilon^{-1} \in \mathcal{U U}^{-1} \cap \Omega\left(X, x_{0}\right) \subseteq h^{-1}\left(F \mathcal{H}\left(y_{0}\right)\right)$ and thus $y_{0} \cdot[\delta]=y_{0} \cdot[\epsilon]$.

Remark 7.11. It is worthwhile to note that when $V$ is path connected

$$
B\left(y_{0} \cdot[\alpha], \mathcal{U}, V\right)=\left\{y_{0} \cdot[\alpha * \xi] \mid \xi \in(\mathcal{P} V)_{\alpha(1)}\right\} .
$$

Thus if $X$ is locally path connected, the construction of $\tilde{X}_{F}$ agrees with the widely used construction of coverings (and generalized coverings [13]) of locally path connected spaces.

By Lemma 7.10, if $p_{F}(y)=x$, the set $y \cdot(\pi X)_{x}=\left\{y \cdot[\alpha] \in \tilde{X}_{F} \mid \alpha \in(\mathcal{P} X)_{x}\right\}$ is open in $\tilde{X}_{F}$. Since $X$ is path connected, $(\mathcal{P} X)_{x}$ is path connected and therefore $y \cdot(\pi X)_{x}$ is path connected.

Proposition 7.12. The path components of $\tilde{X}_{F}$ are the open sets $y \cdot(\pi X)_{x}$.

Proof. If $y \in y_{1} \cdot(\pi X)_{x_{1}} \cap y_{2} \cdot(\pi X)_{x_{2}}$, then $y_{1} \cdot\left[\alpha_{1}\right]=y=y_{2} \cdot\left[\alpha_{2}\right]$ for paths $\alpha_{i}$ satisfying $\alpha_{i}(0)=x_{i}$ and $\alpha_{1}(1)=\alpha_{2}(1)$. We claim that $y_{1} \cdot(\pi X)_{x_{1}}=y_{2} \cdot(\pi X)_{x_{2}}$. If $y_{1} \cdot[\beta] \in y_{1} \cdot(\pi X)_{x_{1}}$ where $\beta(0)=x_{1}$, then

$$
y_{1} \cdot[\beta]=y_{1} \cdot\left[\alpha_{1}\right]\left[\alpha_{1}^{-1} * \beta\right]=y_{2} \cdot\left[\alpha_{2}\right]\left[\alpha_{1}^{-1} * \beta\right]=y_{2} \cdot\left[\alpha_{2} * \alpha_{1}^{-1} * \beta\right]
$$

giving $y_{1} \cdot[\beta] \in y_{2} \cdot(\pi X)_{x_{2}}$. The other inclusion follows similarly.

The general idea of the proof of the following theorem is based on that used by Fischer and Zastrow in [13, Prop. 6.7,6.8] to determine when certain maps have unique path lifting. We first note that if $p_{F}(y)=x$ and $\alpha \in \Omega(X, x)$, the coset $F \mathcal{H}(y)[\alpha] \in$ $F \mathcal{H}(y) \backslash \pi_{1}^{\tau}(X, x)$ is an open neighborhood of $[\alpha]$ in $\pi_{1}^{\tau}(X, x)$ and $h^{-1}(F \mathcal{H}(y)[\alpha])$ is an open neighborhood of $\alpha$ in $\Omega(X, x)$.

Theorem 7.13. If $X$ is locally wep-connected and $F: \mathcal{H} \rightarrow \pi^{\tau} X$ is an open covering morphism of Top-groupoids, then $p_{F}: \tilde{X}_{F} \rightarrow X$ is a semicovering map.

Proof. By Theorem 7.7 and Lemma 7.10, it suffices to show that $p_{F}$ has unique path lifting. Let $f, g: I \rightarrow \tilde{X}_{F}$ be paths such that $p_{F} \circ f=p_{F} \circ g$. We show that either $\{t \in I \mid f(t)=g(t)\}$ is either empty or $I$. By Proposition 7.12, we may assume that $f$ and $g$ have image in a path component $y_{0} \cdot(\pi X)_{x_{0}}$ where $p_{F}\left(y_{0}\right)=x_{0}$. Using Corollary 6.10 , we have $f(t)=y_{0} \cdot\left[\alpha_{t}\right]$ and $g(t)=y_{0} \cdot\left[\beta_{t}\right]$ for locally well-targeted paths 
$\alpha_{t}, \beta_{t} \in(\mathcal{P} X)_{x_{0}}$. The condition $p_{F} \circ f=p_{F} \circ g$ means $\alpha_{t}(1)=\beta_{t}(1)$ for each $t \in I$ and thus $\alpha_{t} * \beta_{t}^{-1} \in \Omega\left(X, x_{0}\right)$. Let $\ell_{t}=\left[\alpha_{t} * \beta_{t}^{-1}\right]$ so that $h^{-1}\left(F \mathcal{H}\left(y_{0}\right) \ell_{t}\right)$ is an open neighborhood of $\alpha_{t} * \beta_{t}^{-1}$ in $\Omega\left(X, x_{0}\right)$. Since $\alpha_{t} * \alpha_{t}^{-1}$ and $\beta_{t} * \beta_{t}^{-1}$ are null-homotopic, there is an open neighborhood $\mathcal{A}_{t}=\bigcap_{j=1}^{n_{t}}\left\langle K_{n_{t}}^{j}, A_{j}^{t}\right\rangle$ of $\alpha_{t}$ and $\mathcal{B}_{t}=\bigcap_{i=1}^{m_{t}}\left\langle K_{m_{t}}^{i}, B_{i}^{t}\right\rangle$ of $\beta_{t}$ in $(\mathcal{P} X)_{x_{0}}$ such that

$$
\left.\left(\left(\mathcal{A}_{t} \mathcal{A}_{t}^{-1}\right) \cup\left(\mathcal{B}_{t} \mathcal{B}_{t}^{-1}\right)\right) \cap \Omega\left(X, x_{0}\right) \subseteq h^{-1}\left(F \mathcal{H}\left(y_{0}\right)\right)\right\}
$$

and

$$
\mathcal{A}_{t} \mathcal{B}_{t}^{-1} \cap \Omega\left(X, x_{0}\right) \subseteq h^{-1}\left(F \mathcal{H}\left(y_{0}\right) \ell_{t}\right) .
$$

Since $\alpha_{t}, \beta_{t}$ are locally well-targeted, there is an open neighborhood $U_{t} \subseteq A_{n_{t}}^{t}$ of $\alpha_{t}(1)$ (resp. $V_{t} \subseteq B_{m_{t}}^{t}$ of $\beta_{t}(1)$ ) such that for each $u \in U_{t}$ (resp. $v \in V_{t}$ ), there is a well-targeted path $\delta \in \mathcal{A}_{t}$ with $\delta(1)=u$ (resp. $\gamma \in \mathcal{B}_{t}$ with $\gamma(1)=v$ ). According to Lemma 7.10, for each $t \in I$,

$$
B\left(y_{0} \cdot\left[\alpha_{t}\right], \mathcal{A}_{t}, U_{t}\right) \text { and } B\left(y_{0} \cdot\left[\beta_{t}\right], \mathcal{B}_{t}, V_{t}\right)
$$

are open neighborhoods of $y_{0} \cdot\left[\alpha_{t}\right]$ and $y_{0} \cdot\left[\beta_{t}\right]$ in $y_{0} \cdot(\pi X)_{x_{0}}$ respectively. Suppose there are $r, s \in I$ such that $y_{0} \cdot\left[\alpha_{r}\right] \neq y_{0} \cdot\left[\beta_{r}\right]$ and $y_{0} \cdot\left[\alpha_{s}\right]=y_{0} \cdot\left[\beta_{s}\right]$. Without loss of generality, we assume $r<s$. Let $z$ be the greatest lower bound of

$$
A=\left\{t \in[r, s] \mid y_{0} \cdot\left[\alpha_{t}\right]=y_{0} \cdot\left[\beta_{t}\right]\right\}=\left\{t \in[r, s] \mid\left[\alpha_{t} * \beta_{t}^{-1}\right] \in F \mathcal{H}\left(y_{0}\right)\right\} .
$$

Since $f$ and $g$ are continuous, there is an $\epsilon>0$ such that $y_{0} \cdot\left[\alpha_{t}\right] \in B\left(y_{0} \cdot\left[\alpha_{z}\right], \mathcal{A}_{z}, U_{z}\right)$ and $y_{0} \cdot\left[\beta_{t}\right] \in B\left(y_{0} \cdot\left[\beta_{z}\right], \mathcal{B}_{z}, V_{z}\right)$ for all $t \in(z-\epsilon, z+\epsilon) \cap[0,1]$. We consider two cases:

(1) If $z \in A$ (equivalently $\left[\alpha_{z} * \beta_{z}^{-1}\right] \in F \mathcal{H}\left(y_{0}\right)$ ), then $r<z \leqslant s$ and $F \mathcal{H}\left(y_{0}\right) \ell_{z}=$ $F \mathcal{H}\left(y_{0}\right)$. Pick any $t_{0} \in(r, z) \cap(z-\epsilon, z)$. We have

$$
y_{0} \cdot\left[\alpha_{t_{0}}\right] \in B\left(y_{0} \cdot\left[\alpha_{z}\right], \mathcal{A}_{z}, U_{z}\right) \text { and } y_{0} \cdot\left[\beta_{t_{0}}\right] \in B\left(y_{0} \cdot\left[\beta_{z}\right], \mathcal{B}_{z}, V_{z}\right)
$$

and therefore $y_{0} \cdot\left[\alpha_{t_{0}}\right]=y_{0} \cdot[\zeta]$ for $\zeta \in \mathcal{A}_{z}$ and $y_{0} \cdot\left[\beta_{t_{0}}\right]=y_{0} \cdot[\eta]$ for $\eta \in \mathcal{B}_{z}$. Since $\zeta(1)=\alpha_{t_{0}}(1)=\beta_{t_{0}}(1)=\eta(1)$, we have

$$
\zeta * \eta^{-1} \in \mathcal{A}_{z} \mathcal{B}_{z}^{-1} \cap \Omega\left(X, x_{0}\right) \subseteq h^{-1}\left(F \mathcal{H}\left(y_{0}\right) \ell_{z}\right)=h^{-1}\left(F \mathcal{H}\left(y_{0}\right)\right)
$$

and $y_{0} \cdot\left[\alpha_{t_{0}}\right]=y_{0} \cdot[\zeta]=y_{0} \cdot[\eta]=y_{0} \cdot\left[\beta_{t_{0}}\right]$. But $t_{0}<z$ and $t_{0} \in A$ contradicting that $z$ is a lower bound for $A$.

(2) If $z \notin A$ (equivalently $\left[\alpha_{z} * \beta_{z}^{-1}\right] \notin F \mathcal{H}\left(y_{0}\right)$ ), then $r \leqslant z<s$ and $F \mathcal{H}\left(y_{0}\right) \ell_{z} \cap$ $F \mathcal{H}\left(y_{0}\right)=\emptyset$. Pick any $t_{0} \in(z, s) \cap(z, z+\epsilon)$ so that, again, $y_{0} \cdot\left[\alpha_{t_{0}}\right]=y_{0} \cdot[\zeta]$ for $\zeta \in$ $\mathcal{A}_{z}$ and $y_{0} \cdot\left[\beta_{t_{0}}\right]=y_{0} \cdot[\eta]$ for $\eta \in \mathcal{B}_{z}$. If $y_{0} \cdot\left[\alpha_{t_{0}}\right]=y_{0} \cdot\left[\beta_{t_{0}}\right]$, then $\left[\zeta * \eta^{-1}\right] \in F \mathcal{H}\left(y_{0}\right)$. But this cannot occur since

$$
\zeta * \eta^{-1} \in \mathcal{A}_{z} \mathcal{B}_{z}^{-1} \cap \Omega\left(X, x_{0}\right) \subseteq h^{-1}\left(F \mathcal{H}\left(y_{0}\right) \ell_{z}\right)
$$

and $F \mathcal{H}\left(y_{0}\right) \ell_{z} \cap F \mathcal{H}\left(y_{0}\right)=\emptyset$. Thus $y_{0} \cdot\left[\alpha_{t}\right] \neq y_{0} \cdot\left[\beta_{t}\right]$ for each $t \in[z, s) \cap[z, z+\epsilon)$. That any $y \in(z, s) \cap(z, z+\epsilon)$ is a lower bound for $A$ greater than $z$ gives the desired contradiction.

\subsection{Proof of Theorem 7.1}

To complete the proof of Theorem 7.1, we define the inverse equivalence

$$
\mathscr{S}: \operatorname{OCovMor}\left(\pi^{\tau} X\right) \rightarrow \operatorname{SCov}(X)
$$


as $\mathscr{S}(F)=p_{F}: \tilde{X}_{F} \rightarrow X$ for an open covering morphism $F: \mathcal{H} \rightarrow \pi^{\tau} X$. Following Proposition 7.3, let $\mathscr{S}(K)=O b(K): \tilde{X}_{F} \rightarrow \tilde{X}_{F^{\prime}}$ for a morphism

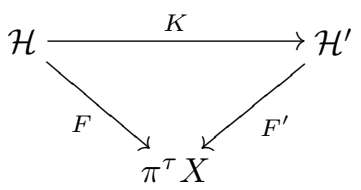

in $\operatorname{OCovMor}\left(\pi^{\tau} X\right)$.

Suppose $p: Y \rightarrow X$ is a semicovering of $X$ and $F=\pi^{\tau} p: \pi^{\tau} Y \rightarrow \pi^{\tau} X$. Certainly $\mathscr{S}(F)=p_{F}: \tilde{X}_{F} \rightarrow X$ and $p$ agree as functions since both are $O b(\pi p)$. To check that the topologies of $Y$ and $\tilde{X}_{F}$ agree, consider the following diagram.

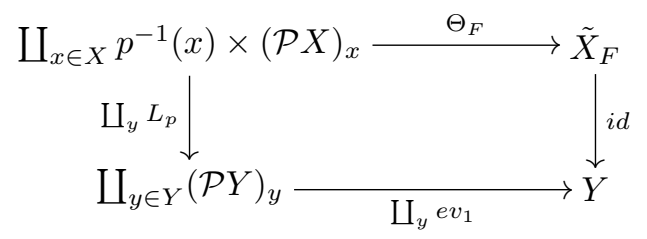

The left vertical map takes $(y, \alpha) \in p^{-1}(x) \times(\mathcal{P} X)_{x}$ to the lift $\tilde{\alpha}_{y} \in(\mathcal{P} Y)_{y}$ and is a homeomorphism since $p$ has continuous lifting of paths. The bottom horizontal map is evaluation at 1 on each summand and is quotient by Propositions 6.12 and 6.2. Since $\Theta_{F}$ is quotient by definition, $i d: \tilde{X}_{F} \rightarrow Y$ is continuous and open. One could equally have chosen to apply Lemma 2.5 to draw this conclusion. Suppose $p^{\prime}: Y^{\prime} \rightarrow X$ is another semicovering of $X, F^{\prime}=\pi^{\tau} p^{\prime}$, and $f: Y \rightarrow Y^{\prime}$ is a map such that $p^{\prime} \circ f=p$. Then $\mathscr{S}\left(\pi^{\tau} f\right)$ is the map

$$
f=O b\left(\pi^{\tau} f\right): \tilde{X}_{F} \rightarrow \tilde{X}_{F^{\prime}}
$$

since $\tilde{X}_{F}=Y$ and $\tilde{X}_{F^{\prime}}=Y^{\prime}$. Thus $\mathscr{S} \circ \pi^{\tau} \simeq I d$ where the components of the natural isomorphism are identity maps.

Now suppose $F: \mathcal{H} \rightarrow \pi^{\tau} X$ is an open covering morphism. By Theorem 7.13, $\mathscr{S}(F)=p_{F}: \tilde{X}_{F} \rightarrow X$ is a semicovering which induces an open covering morphism $\pi^{\tau} p_{F}: \pi^{\tau} \tilde{X}_{F} \rightarrow \pi^{\tau} X$. We define a functor $N_{F}: \mathcal{H} \rightarrow \pi^{\tau} \tilde{X}_{F}$ such that $\operatorname{Ob}\left(N_{F}\right)=$ $I d_{O b(\mathcal{H})}$. If $h \in \mathcal{H}\left(y_{1}, y_{2}\right)$ and $F(h)=[\alpha] \in \pi^{\tau} X\left(x_{1}, x_{2}\right)$, let

$$
N_{F}(h)=\left[\tilde{\alpha}_{y_{1}}\right] \in \pi^{\tau} \tilde{X}_{F}\left(y_{1}, y_{2}\right)
$$

be the homotopy class of the unique lift of $\alpha$ with respect to $p_{F}$. Certainly, $\tilde{\alpha}_{y_{1}}(1)=y_{2}$ since the description of lifts of paths in 7.5 gives that $\tilde{\alpha}_{y_{1}}(1)=y_{1} \cdot F(h)=y_{2}$. Since

$$
\pi^{\tau} p_{F} \circ N_{F}=F
$$

$N_{F}$ is an open covering morphism by Proposition 5.2. But $O b\left(N_{F}\right)$ is the identity on objects and so $N_{F}: \mathcal{H} \rightarrow \pi^{\tau} \tilde{X}_{F}$ is an isomorphism of Top-groupoids. It suffices check that the $N_{F}$ form a natural isomorphism $I d \simeq \pi^{\tau} \circ \mathscr{S}$, i.e., that the following diagram of Top-functors commutes for a morphism $K: \mathcal{H} \rightarrow \mathcal{H}^{\prime}$ of open covering 
morphisms $F$ and $F^{\prime}: \mathcal{H}^{\prime} \rightarrow \pi^{\tau} X$.

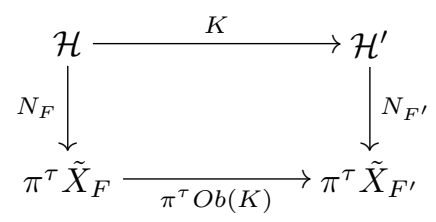

Certainly, the diagram commutes on objects. Suppose $g \in \mathcal{H}\left(y_{1}, y_{2}\right)$ and $F(g)=[\alpha]$ so that $N_{F}(g)=\left[\tilde{\alpha}_{y_{1}}\right]$ as above. Then $\pi^{\tau} \operatorname{Ob}(K)\left(\left[\tilde{\alpha}_{y_{1}}\right]\right)$ is the homotopy class $\left[\tilde{\alpha}_{K\left(y_{1}\right)}\right]$ of the unique lift of $\alpha$ with respect to $p_{F^{\prime}}: \tilde{X}_{F^{\prime}} \rightarrow X$ starting at $K\left(y_{1}\right)$. The definition of $N_{F^{\prime}}$ gives $N_{F^{\prime}}(K(g))=\left[\tilde{\alpha}_{K\left(y_{1}\right)}\right]$ since $F^{\prime}(K(g))=F(g)=[\alpha]$ and $K(g) \in$ $\mathcal{H}^{\prime}\left(K\left(y_{1}\right), K\left(y_{2}\right)\right)$.

This completes the proof of Theorem 7.1.

\subsection{Enriched monodromy}

In this section, we offer an alternative classification of semicoverings in terms of monodromy.

Definition 7.14. The monodromy of a map $p: Y \rightarrow X$ with continuous lifting of paths and homotopies is the functor $\mathscr{M} p: \pi X \rightarrow$ Set which takes a point $x \in X$ to the fiber $p^{-1}(x)$ and a class $[\alpha] \in \pi X\left(x_{1}, x_{2}\right)$ to the function $p^{-1}\left(x_{1}\right) \rightarrow p^{-1}\left(x_{2}\right)$, $y \mapsto y \cdot[\alpha]=\tilde{\alpha}_{y}(1)$.

The monodromy of a semicovering becomes an enriched functor when we use $\pi^{\tau} X$ and view Set as a Top-category by giving each set the discrete topology and endowing $\operatorname{Set}(A, B)$ with the topology of pointwise convergence (which is equivalent to the compact-open topology).

Theorem 7.15. The monodromy $\mathscr{M} p: \pi^{\tau} X \rightarrow$ Set of a semicovering $p: Y \rightarrow X$ is a Top-functor. Moreover, $\mathscr{M}: \operatorname{SCov}(X) \rightarrow \operatorname{TopFunc}\left(\pi^{\tau} X\right.$, Set $)$ is a functor.

Proof. Suppose $p\left(y_{i}\right)=x_{i}, i=1,2$. The set $\left\{[\alpha] \in \pi X\left(x_{1}, x_{2}\right) \mid y_{1} \cdot[\alpha]=y_{2}\right\}$ is open in $\pi^{\tau} X\left(x_{1}, x_{2}\right)$ since it is the image of the open embedding $\pi^{\tau} p: \pi^{\tau} Y\left(y_{1}, y_{2}\right) \rightarrow$ $\pi^{\tau} X\left(x_{1}, x_{2}\right)$ (Recall Theorem 5.5). Additionally, each fiber $p^{-1}(x)$ is discrete. Therefore each action map

$$
p^{-1}\left(x_{1}\right) \times \pi^{\tau} X\left(x_{1}, x_{2}\right) \rightarrow p^{-1}\left(x_{2}\right),(y,[\alpha]) \mapsto y \cdot[\alpha]
$$

is continuous. Since discrete spaces are locally compact Hausdorff, the adjoint map

$$
\mathscr{M} p: \pi^{\tau} X\left(x_{1}, x_{2}\right) \rightarrow \operatorname{Set}\left(p^{-1}\left(x_{1}\right), p^{-1}\left(x_{2}\right)\right) \text { where } \mathscr{M} p([\alpha])(y)=y \cdot[\alpha]
$$

is continuous. Thus $\mathscr{M} p: \pi^{\tau} X \rightarrow$ Set is a Top-functor. A morphism $f: Y \rightarrow Y^{\prime}$ of semicoverings $p: Y \rightarrow X$ and $p^{\prime}: Y^{\prime} \rightarrow X$ induces the natural transformation $\mathscr{M} f: \mathscr{M} p \rightarrow \mathscr{M} p^{\prime}$ with components $f: p^{-1}(x) \rightarrow\left(p^{\prime}\right)^{-1}(x)$.

Corollary 7.16. For each $x_{0} \in X$, monodromy of a semicovering $p: Y \rightarrow X$ restricts to a continuous group homomorphism $\pi_{1}^{\tau}\left(X, x_{0}\right) \rightarrow \operatorname{Homeo}\left(p^{-1}\left(x_{0}\right)\right)$.

It is well-known that the category of covering morphisms $\operatorname{CovMor}(\mathcal{G})$ of a groupoid $\mathcal{G}$ is naturally equivalent to the functor category $\operatorname{Func}(\mathcal{G}$, Set $)$ which is often 
referred to as the category of representations of $\mathcal{G}$ [18, Prop. 30]. In particular, a covering morphism $F: \mathcal{H} \rightarrow \mathcal{G}$ corresponds to the functor $\mathscr{R} F: \mathcal{G} \rightarrow$ Set given by $\mathscr{R} F(x)=O b(F)^{-1}(x)$ on object $x \in O b(\mathcal{G})$ and for $g \in \mathcal{G}\left(x_{1}, x_{2}\right), \mathscr{R} F(g)$ is the function $O b(F)^{-1}\left(x_{1}\right) \rightarrow O b(F)^{-1}\left(x_{2}\right), y \mapsto t\left(\tilde{g}_{y}\right)$ where $t: \mathcal{H} \rightarrow O b(\mathcal{H})$ is the target map of $\mathcal{H}$. The inverse $\mathscr{R}^{-1}: \operatorname{Func}(\mathcal{G}$, Set $) \rightarrow \operatorname{CovMor}(\mathcal{G})$ may be described as follows: Given a functor $M: \mathcal{G} \rightarrow$ Set, let $\mathcal{H}$ be the groupoid with object set $\bigcup_{x \in O b(\mathcal{G})} M(x)$ and if $y_{i} \in M\left(x_{i}\right), i=1,2$, then

$$
\mathcal{H}\left(y_{1}, y_{2}\right)=\left\{g \in \mathcal{G}\left(x_{1}, x_{2}\right) \mid M(g)\left(y_{1}\right)=y_{2}\right\} .
$$

The functor $\mathscr{R}^{-1} M: \mathcal{H} \rightarrow \mathcal{G}$ taking $y \in M(x)$ to $x$ and which is the inclusion on hom-sets is the corresponding covering morphism.

Theorem 7.17. For a Top-groupoid $\mathcal{G}$, there is an equivalence of categories

$$
\mathscr{R}: \operatorname{OCovMor}(\mathcal{G}) \rightarrow \operatorname{TopFunc}(\mathcal{G}, \text { Set }) .
$$

Proof. We construct $\mathscr{R}$ and its inverse so that when topological structure is forgotten we retain the constructions in above equivalence. Note that a subbasis set for the topology of

$$
\text { Set }\left(O b(F)^{-1}\left(x_{1}\right), O b(F)^{-1}\left(x_{2}\right)\right)
$$

is of the form $\left\langle\left\{y_{1}\right\},\left\{y_{2}\right\}\right\rangle$. Thus if $F: \mathcal{H} \rightarrow \mathcal{G}$ is an open covering morphism, then

$$
\mathscr{R} F: \mathcal{G}\left(x_{1}, x_{2}\right) \rightarrow \operatorname{Set}\left(O b(F)^{-1}\left(x_{1}\right), O b(F)^{-1}\left(x_{2}\right)\right)
$$

is continuous since each set

$$
\begin{aligned}
(\mathscr{R} F)^{-1}\left(\left\langle\left\{y_{1}\right\},\left\{y_{2}\right\}\right\rangle\right) & =\left\{g \in \mathcal{G}\left(x_{1}, x_{2}\right) \mid t\left(\tilde{g}_{y_{1}}\right)=y_{2}\right\} \\
& =\operatorname{Im}\left(F: \mathcal{H}\left(y_{1}, y_{2}\right) \rightarrow \mathcal{G}\left(x_{1}, x_{2}\right)\right)
\end{aligned}
$$

is open in $\mathcal{G}\left(x_{1}, x_{2}\right)$. Let $\mathcal{G}$ be a Top-groupoid and $M: \mathcal{G} \rightarrow$ Set be a Top-functor. We define $\mathcal{H}$ and $\mathscr{R}^{-1} M: \mathcal{H} \rightarrow \mathcal{G}$ as above but give $\mathcal{H}\left(y_{1}, y_{2}\right)$ the subspace topology of $\mathcal{G}\left(x_{1}, x_{2}\right)$ when $y_{i} \in M\left(x_{i}\right)$. This makes $\mathcal{H}$ a Top-groupoid and $\mathscr{R}^{-1} M$ a Top-functor. Since $M: \mathcal{G}\left(x_{1}, x_{2}\right) \rightarrow \operatorname{Set}\left(M\left(x_{1}\right), M\left(x_{2}\right)\right)$ is continuous and each $M\left(x_{i}\right)$ is discrete, $\mathcal{H}\left(y_{1}, y_{2}\right)$ is open in $\mathcal{G}\left(x_{1}, x_{2}\right)$ whenever $y_{i} \in M\left(x_{i}\right)$. Thus $\mathscr{R}^{-1} M$ is open.

Note that $\mathscr{M}=\mathscr{R} \circ \pi^{\tau}$ where $\pi^{\tau}$ is the equivalence of Theorem 7.1. Thus semicoverings are also classified by monodromy.

Theorem 7.18. Let $X$ be a locally wep-connected space. Monodromy

$$
\mathscr{M}: \operatorname{SCov}(X) \rightarrow \operatorname{TopFunc}\left(\pi^{\tau} X, \operatorname{Set}\right)
$$

is an equivalence of categories.

\subsection{Semicoverings and $G$-sets}

The classification of coverings of a path connected, locally path connected, semilocally 1-connected space $X$ may also be expressed as an equivalence of categories

$$
\operatorname{Cov}(X) \simeq \pi_{1}\left(X, x_{0}\right) \text { Set },
$$

where $\pi_{1}\left(X, x_{0}\right)$ Set is the category of sets with right $\pi_{1}\left(X, x_{0}\right)$ actions. We obtain a similar formulation of the classification of semicoverings. 
Let $G$ be a topological group and $A$ be a set with the discrete topology. A right action of $G$ on $A$ is a continuous group action $A \times G \rightarrow A$. For each $x \in A$, the restriction $G \rightarrow x G=\{x g \in A \mid g \in G\}$ of the action induces a continuous bijection from the quotient right coset space $H \backslash G$ (where $H$ is the stabilizer at $x$ ) to $x G$. Thus continuity of the action of $G$ on discrete $A$ is equivalent to all stabilizer subgroups being open in $G$. A right $G$-set is a discrete set $A$ with a right action of $G$. This agrees with the usual notion of right $G$-set when $G$ is discrete. The category of right $G$-sets and $G$-maps $\psi: A \rightarrow B$ is denoted $G$ Set. Note that $G$ Set is naturally equivalent to $\operatorname{TopFunc}(G, \operatorname{Set})$ when $G$ is viewed as a Top-groupoid with a single object.

A right $G$-set $A$ is transitive if $A=x G$ for some $x \in A$. In this case, $A$ may be identified in $G$ Set with the discrete right coset space $H \backslash G=\{H g \mid g \in G\}$ where, again, $H$ is the stabilizer at $x$. Define the orbit category of the topological group $G$ to be the full subcategory $\mathscr{O}_{G}$ of $G$ Set generated by transitive right $G$-sets. Note that $\mathscr{O}_{G}$ is equivalent to the full subcategory of $G$ Set generated by $G$-sets $H \backslash G$ where $H$ is an open subgroup of $G$. The well-known theory for discrete groups extends to the non-discrete case giving that the objects of $\mathscr{O}_{G}$ correspond to open subgroups of $G$ and isomorphism classes correspond to conjugacy classes of open subgroups of $G$.

Theorem 7.19. Let $X$ be a locally wep-connected space and $x_{0} \in X$.

1. There is an equivalence $\mathbf{S} \operatorname{Cov}(X) \rightarrow \pi_{1}^{\tau}\left(X, x_{0}\right)$ Set of categories taking a semicovering $p: Y \rightarrow X$ to the fiber $p^{-1}\left(x_{0}\right)$ with action

$$
p^{-1}\left(x_{0}\right) \times \pi_{1}^{\tau}\left(X, x_{0}\right) \rightarrow p^{-1}\left(x_{0}\right) \text { given by }(y,[\alpha]) \mapsto \tilde{\alpha}_{y}(1) .
$$

2. The equivalence in 1. restricts to an equivalence of categories $\mathbf{S C o v}_{\mathbf{0}}(X) \simeq$ $\mathscr{O}_{\pi_{1}^{\tau}\left(X, x_{0}\right)}$.

Proof. 1. The desired equivalence is the composition of equivalences:

$$
\operatorname{SCov}(X) \simeq \operatorname{TopFunc}\left(\pi^{\tau} X, \operatorname{Set}\right) \simeq \operatorname{TopFunc}\left(\pi_{1}^{\tau}\left(X, x_{0}\right), \operatorname{Set}\right) \simeq \pi_{1}^{\tau}\left(X, x_{0}\right) \text { Set. }
$$

The first equivalence comes from monodromy (Theorem 7.18). The vertex group inclusion $\pi_{1}^{\tau}\left(X, x_{0}\right) \rightarrow \pi^{\tau} X$ is a Top-equivalence since $X$ is path connected and translations in $\pi^{\tau} X$ are continuous. This gives the second equivalence on enriched functor categories. The last equivalence is an observation made in the second paragraph of this section.

2. The equivalence from 1. restricts to an equivalence $\mathbf{S C o v}_{\mathbf{0}}(X) \simeq \mathscr{O}_{\pi_{1}^{\tau}\left(X, x_{0}\right)}$ due to the fact that given a semicovering $p: Y \rightarrow X, Y$ is path connected if and only if the action $p^{-1}\left(x_{0}\right) \times \pi_{1}^{\tau}\left(X, x_{0}\right) \rightarrow p^{-1}\left(x_{0}\right)$ is transitive.

Corollary 7.20. Let $X$ be a locally wep-connected space and $x_{0} \in X$. There is a Galois correspondence between the equivalence classes of connected semicoverings of $X$ and conjugacy classes of open subgroups of $\pi_{1}^{\tau}\left(X, x_{0}\right)$.

Corollary 7.21. If $X$ is locally wep-connected and $x_{0} \in X$, then $X$ has a universal semicovering if and only if there is an open subgroup $S$ in $\pi_{1}^{\tau}\left(X, x_{0}\right)$ such that for any other open subgroup $H$ of $\pi_{1}^{\tau}\left(X, x_{0}\right)$, there is a $g \in \pi_{1}^{\tau}\left(X, x_{0}\right)$ such that $g S g^{-1} \subseteq H$. 


\section{References}

[1] S. Ardanza-Trevijana, L.-J. Hernandez-Paricio, Fundamental progroupoid and bundles with a structural category, Topology Appl. 92 (1999) 85-99.

[2] W. Bogley, A. Sieradski, Universal path spaces, http://people. oregonstate. edu/ ${ }^{\sim}$ bogleyw/research/ups.pdf.

[3] J. Brazas, The topological fundamental group and free topological groups, Topology Appl. 158 (2011) 779-802.

[4] J. Brazas, The fundamental group as topological group, preprint, arXiv:1009.3972v4, 2011.

[5] N. Brodskiy, J. Dydak, B. Labuz, A. Mitra, Covering maps for locally path connected spaces, preprint, arXiv:0801.4967v3, 2008.

[6] R. Brown, Topology and Groupoids, Booksurge PLC, 2006.

[7] R. Brown, J.P.L. Hardy, Topological groupoids I: universal constructions, Math. Nachr. 71 (1976) 273-286.

[8] R. Brown, Function spaces and product topologies, Quart. J. Math. (2) 15 (1964) 238-250.

[9] J. Calcut, J. McCarthy, Discreteness and homogeneity of the topological fundamental group, Topology Proc. 34 (2009) 339-349.

[10] P. Fabel, Multiplication is discontinuous in the hawaiian earring group (with the quotient topology), Bulletin Pol. Acad. Sci. 59 (1) (2011) 77-83.

[11] P. Fabel, Compactly generated quasitopological homotopy groups with discontinuous multiplication, Topology Proc., to appear.

[12] H. Fischer, D. Repovs, Z. Virk, A. Zastrow, On semilocally simply connected spaces, Topology Appl. 158 (3) (2011) 397-408.

[13] H. Fischer, A. Zastrow, Generalized universal covering spaces and the shape group, Fund. Math. 197 (2007) 167-196.

[14] R. Fox, Shape theory and covering spaces, Vol. 375 of Lecture Notes in Mathematics, Springer Verlag, 1974.

[15] A. Hatcher, Algebraic Topology, Cambridge University Press, 2002.

[16] M. Henriksen, R.G. Woods, Separate verses joint continuity: A tale of four topologies, Topology Appl. 97 (1999) 175-205.

[17] L.-J. Hernandez-Paricio, Fundamental pro-groupoids and covering projections, Fund. Math. 156 (1998) 1-33.

[18] P. Higgins, Notes on categories and groupoids, Vol. 32, Van Nostrand Reinhold Co. London, 1971, also: Reprints in Theory Appl. Categories No. 7 (2005) pp 1-195.

[19] P. Hilton, S. Wylie, Homology Theory: An introduction to algebraic topology, Cambridge University Press, 1960.

[20] G. Kelly, Basic concepts of enriched category theory, Vol. 64 of London Math. Soc. Lec. Notes Series, Cambridge University Press, 1982, also: Reprints in Theory Appl. Categories 10 (2005). 
[21] S. Kocak, On the fundamental theorem of overlays, Note. Mat. 10 (1990) 355362.

[22] S. Lubkin, Theory of covering spaces, Trans. Amer. Math. Soc. 104 (1962) 205-238.

[23] J.P. May, A Concise Course in Algebraic Topology, Chicago Lectures in Mathematics, 1999.

[24] P.A. Smith, The complex of a group relative to a set of generators. Part I, Ann. of Math. 54 (1951) 371-402.

[25] E. Spanier, Algebraic Topology, McGraw-Hill, 1966.

Jeremy Brazas jtv5@unh.edu

Department of Mathematics and Statistics, University of New Hampshire, 33 Academic Way, Durham, NH, 03824, USA 